\title{
Mass Selective Axial Ion Ejection from a Linear Quadrupole Ion Trap
}

\author{
F. A. Londry and James W. Hager \\ MDS SCIEX, Concord, Ontario, Canada
}

\begin{abstract}
The electric fields responsible for mass-selective axial ejection (MSAE) of ions trapped in a linear quadrupole ion trap have been studied using a combination of analytic theory and computer modeling. Axial ejection occurs as a consequence of the trapped ions' radial motion, which is characterized by extrema that are phase-synchronous with the local RF potential. As a result, the net axial electric field experienced by ions in the fringe region, over one RF cycle, is positive. This axial field depends strongly on both the axial and radial ion coordinates. The superposition of a repulsive potential applied to an exit lens with the diminishing quadrupole potential in the fringing region near the end of a quadrupole rod array can give rise to an approximately conical surface on which the net axial force experienced by an ion, averaged over one RF cycle, is zero. This conical surface has been named the cone of reflection because it divides the regions of ion reflection and ion ejection. Once an ion penetrates this surface, it feels a strong net positive axial force and is accelerated toward the exit lens. As a consequence of the strong dependence of the axial field on radial displacement, trapped thermalized ions can be ejected axially from a linear ion trap in a mass-selective way when their radial amplitude is increased through a resonant response to an auxiliary signal. (J Am Soc Mass Spectrom 2003, 14, 1130-1147) (C) 2003 American Society for Mass Spectrometry
\end{abstract}

I ons trapped within a linear quadrupole ion trap can be extracted mass selectively either axially $[1,2]$ or radially [3]. Both approaches yield high quality mass spectra and offer advantages over conventional three-dimensional ion traps, such as greater ion capacity, higher trapping efficiencies, less mass discrimination, and reduced effects of space charge $[1,3]$. In certain cases, linear ion trap mass spectrometers can be incorporated into the ion path of triple quadrupoles yielding an instrument that combines the strengths of both platforms [1, 4]. Ions are trapped radially in these devices by the RF quadrupole field and axially by static DC potentials at the ends of the quadrupole rod array, in contrast to conventional Paul traps in which ions are trapped by a three-dimensional RF quadrupole field. Radial mass-selective ion ejection occurs when the RF voltage is ramped in the presence of a sufficiently intense auxiliary AC voltage. The auxiliary AC resonance-ejection voltage is applied radially and the ions emerge from the linear ion trap through slots cut in the quadrupole rods [3]. Radial ejection requires that the RF field be of high quality over the entire length of the ion trap [3] in order to preserve mass spectral resolution, since resolution depends on the fidelity of the secular frequency of the trapped ions. Thus, very high mechanical precision is required in fabrication of the quadru-

Published online August 11, 2003

Address reprint requests to F. A. Londry, MDX SCIEX, 71 Four Valley Drive, Concord, Ontario L4K 4V8, Canada. E-mail: Flondry@nexicom.net pole rods in order to maintain the same secular frequency over the length of the device. Of course, the greater the length of the linear ion trap, the more difficult it is to maintain the high degree of mechanical precision. In addition, considerable care must be taken to ensure that the ions that are intended to be ejected radially from the linear ion trap are isolated as much as possible from the ends of the linear quadrupole device due to the fringing field effects of the termination of the RF fields [3]. These fringing fields, positioned at both ends of the quadrupole, are most often deleterious to RF/DC quadrupole performance [5] as well as radial ejection from a linear ion trap [3]. In the fringing region, the diminution of the quadrupole field, the increased significance of higher-order terms in the multipole expansion of the potential and coupling of radial and axial fields lead to significant changes in the secular frequency [6,7] and thus ejection at unexpected stability coordinates.

Mass-selective axial ejection (MSAE) of ions from linear quadrupole ion traps takes advantage of the RF fringing fields to convert radial ion excitation into axial ion ejection [1, 2] in a manner analogous to resolving RF-only mass spectrometers [8, 9]. Trapped ions are given some degree of radial excitation via a resonance excitation process, and in the exit fringing-field, this radial excitation results in additional axial ion kinetic energy that can overcome the exit DC barrier [1, 2].

MSAE of ions from a linear quadrupole ion trap has been shown to add high-sensitivity and high-resolution 
capabilities to traditional RF/DC mass-filters [1, 4]. Trapped, thermalized ions can be ejected axially in a mass-selective way by ramping the amplitude of the RF drive, to bring ions of increasingly higher $\mathrm{m} / \mathrm{z}$ into resonance with a single-frequency dipolar auxiliary signal, applied between two opposing rods. In response to the auxiliary signal, ions gain radial amplitude until they are ejected axially or neutralized on the rods [2]. In general, the radial excitation voltage is lower than that used to perform mass-selective radial ejection [3] since the goal is provide a degree of radial excitation rather than radial ejection.

In this work, a combination of analytic theory and computer modeling has been used to study the axial forces that influence ion trajectories in the fringing region at the end of a linear quadrupole ion trap and to elucidate the process of mass-selective axial ejection. Initially, analytic approximations are used to obtain a closed-form expression, which offers insight into the salient characteristics of the net axial force experienced by ions in the fringing region that make MSAE a practical analytic technique. Subsequently, fields obtained by more rigorous, numerical, methods are used to confirm the validity of these approximations and to demonstrate the technique. Finally, simulated massintensity signals are compared with experiment.

\section{Methods}

In this work, a theory is developed, which provides a basis for understanding the process of mass-selective axial ejection (MSAE) from a linear quadrupole ion trap. Subsequently, a trajectory simulator, described in the Appendix, is used to test the validity and the range of applicability of the theory. In addition, numerical methods are used to perform realistic trajectory calculations of ions subject to MSAE and the results are used to relate concepts developed theoretically to mass-spectral characteristics. Finally, mass-intensity signals, obtained through simulation are compared with experimental data.

Before the theory is presented, the trajectory calculations are explained briefly and the experimental apparatus is described. Throughout this paper, a frequency of $1.0 \mathrm{MHz}$ was used exclusively for the quadrupole RF drive.

\section{Trajectory Calculations}

A general description of the trajectory calculator has been provided in the Appendix. In general, trajectories were calculated by integrating an equation of motion with three distinct terms.

$$
\frac{\mathrm{d}^{2} \vec{r}}{\mathrm{~d} t^{2}}=\frac{e}{m}\left[V_{\text {quad }} \vec{E}_{\text {quad }}+U_{\text {lens }} \vec{E}_{\text {lens }}+V_{\text {dipolar }} \vec{E}_{\text {dipolar }}\right]
$$

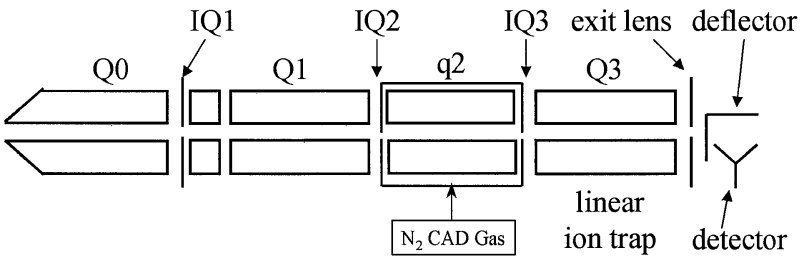

Figure 1. Essential elements of the ion path of the Q TRAP instrument.

where $\vec{r}$ is the position vector of a singly charged positive ion of mass $m$ and $e$ is the electronic charge. $V_{\text {quad }}$ is the amplitude of the quadrupole $R F$ drive, $U_{\text {lens }}$ is the DC potential applied to the exit lens and $V_{\text {dipolar }}$ is the amplitude of the dipolar auxiliary RF signal applied between the $x$-rods. The electric fields, $\vec{E}_{\text {quad }}$, $\vec{E}_{\text {lens }}$ and $\vec{E}_{\text {dipolar }}$ were obtained by numerical methods described in the Appendix for the final $17 \mathrm{~mm}$ of a round-rod quadrupole array followed by a wire-mesh covered exit-lens $3 \mathrm{~mm}$ distant from the ends of the rods yielding an overall axial dimension of $20 \mathrm{~mm}$. The model was terminated in the $x-y$ plane at the end opposite the lens by a plane of even symmetry. To reduce field distortions imposed by this boundary condition to inconsequential levels, the axial dimension of the numerical models was chosen greater than four times the field radius $r_{0}$. The minimum distance between opposing rods was $8.34 \mathrm{~mm}$ and the $r / r_{0}$ ratio was 1.126. Specifically, $\vec{E}_{\text {quad }}$ was obtained by differentiating a numerical solution of the Laplace equation, which was obtained with $\pm 1 \mathrm{~V}$ applied to opposing rods in quadrupole fashion with the exit lens grounded. Similarly, $\vec{E}_{\text {lens }}$ was obtained with the rods grounded and one volt applied to the exit lens. Likewise, $\vec{E}_{\text {dipolar }}$ was obtained with $\pm 1 \mathrm{~V}$ applied to the opposing $x$-rods, with the $y$-rods and exit lens at ground.

In order to make a more direct comparison with the theory, in some cases the first term of Eq 1 was replaced by the gradient of an analytic approximation of the quadrupole potential in the fringing region, $\Phi_{\mathrm{FF}}$, vide infra.

$$
\frac{\mathrm{d}^{2} \vec{r}}{\mathrm{~d} t^{2}}=\frac{e}{m}\left[-\nabla \Phi_{F F}+U_{\text {lens }} \vec{E}_{\text {lens }}+V_{\text {dipolar }} \vec{E}_{\text {dipolar }}\right]
$$

\section{Experimental}

The experimental apparatus was a prototype version of the Q TRAP instrument (Applied Biosystems/MDS SCIEX, Toronto, Canada) with a Q-q- $\mathrm{Q}_{\text {linear in trap }}$ arrangement. Significant elements of the ion path are shown in Figure 1. The ion path was based on that of a triple quadrupole mass spectrometer ion path with the final quadrupole rod array configured to operate as a conventional RF/DC mass filter or a linear ion trap with mass-selective axial ejection. Ions, generated by a pneumatically assisted electrospray ion source, travelled 
through curtain gas and differential pumping regions into an RF-only quadrupole ion guide (Q0) within a chamber maintained at approximately $6 \times 10^{-3}$ torr. The Q0 rods were capacitively coupled to the $1 \mathrm{MHz}$ Q1 drive RF voltage. An additional differential pumping aperture, IQ1, separated the Q0 chamber and the analyzer chamber. A short RF-only Brubaker lens, located in front of the Q1 RF/DC quadrupole mass spectrometer, was coupled capacitively to the Q1 drive RF power supply.

The collision cell (q2) was an enclosed LINAC [10] quadrupole array with the IQ2 and IQ3 lenses located at either end. Nitrogen gas was used as the collision gas. Gas pressures within q2 were calculated from the conductance of IQ2 and IQ3 and the pumping speed of the turbo molecular pumps. Typical operating pressures were about $5 \times 10^{-3}$ torr in q2 and $3.5 \times 10^{-5}$ torr in Q3. The RF voltage used to drive the collision cell rods was transferred from the $1.0 \mathrm{MHz} \mathrm{Q} 3 \mathrm{RF}$ power supply through a capacitive coupling network.

The Q3 quadrupole rod array was mechanically identical to Q1. Two additional lenses were located downstream of Q3, the first, a mesh covered 8-mm aperture, the second, a clear 8-mm diameter aperture. The mesh-covered lens is referred to as the exit lens and the clear aperture lens is referred to as the deflector. Typically, the deflector lens was operated at about 200 $\mathrm{V}$ attractive with respect to the exit lens in order to draw ions away from the Q3 ion trap toward the ion detector.

Ions were detected via an ETP (Sydney, Australia) discrete dynode electron multiplier, operated in pulse counting mode, with the entrance floated to $-6 \mathrm{kV}$ for positive ion detection and $+4 \mathrm{kV}$ for detection of negative ions.

The source for the auxiliary AC signal was a HP 33120A signal generator (Hewlett Packard, Palo Alto, CA) capable of variable frequency and voltage operation. The auxiliary AC voltage was applied to Q3 in a dipolar fashion via a centre-tap bipolar toroidal transformer and its amplitude was ramped proportionally to mass. The ions trapped within the Q3 linear ion trap were excited resonantly at $\beta=0.76$ by a $380 \mathrm{kHz}$ signal, corresponding to $(a, q)=(0,0.84)$.

The reserpine solution used in these experiments was obtained from Sigma-Aldrich Canada Ltd. and was used at a concentration of 1 picomole per microlitre.

\section{Theory}

The behavior of ions, in response to a combination of RF and DC quadrupole potentials, has been described thoroughly by Dawson [11]. For completeness and continuity, some of that theory has been reproduced herein.

In the central portion of a linear ion trap where end effects are negligible, the two-dimensional quadrupole potential can be written as

$$
\Phi_{2 \mathrm{D}}-\varphi_{0} \frac{x^{2}-y^{2}}{r_{0}^{2}}
$$

where $2 r_{0}$ is the shortest distance between opposing rods and $\varphi_{0}$ is the electric potential, measured with respect to ground, applied with opposite polarity to each of the two poles. Traditionally, $\varphi_{0}$ has been written as a linear combination of DC and RF components as

$$
\Phi_{0}=U-V \cos \Omega t
$$

where $\Omega$ is the angular frequency of the RF drive.

In response to the potential described by $\mathrm{Eq} 3$, the equation of motion for a singly charged positive ion of mass $m$ is

$$
\frac{\mathrm{d}^{2} \vec{r}}{\mathrm{~d} t^{2}}=\frac{e}{m} \nabla \Phi_{2 \mathrm{D}}
$$

where $e$ is the electronic charge. With the substitution of the dimensionless parameter

$$
\xi=\frac{\Omega t}{2}
$$

Eq 5 can be cast in Mathieu form as

$$
\frac{\mathrm{d}^{2} u}{\mathrm{~d} \xi^{2}}+\left(a_{\mathrm{u}}-2 q_{\mathrm{u}} \cos 2 \xi\right) u=0
$$

where $u$ can be either $x$ or $y$ and

$$
a_{u}= \pm \frac{8 e U}{m r_{0}^{2} \Omega^{2}}
$$

and

$$
q_{u}= \pm \frac{4 e V}{m r_{0}^{2} \Omega^{2}}
$$

where the + and - signs correspond to $u=x$ and $u=$ $y$, respectively.

Consider motion in the $x$-direction and assume, following Dehmelt [12], that ion motion can be approximated by a slowly varying high amplitude secular motion $X$ and a rapidly varying low amplitude micromotion $\delta_{x}$ such that

$$
x=X+\delta_{x}
$$

where $\delta_{x}<<X$ and $\frac{\mathrm{d}^{2} \delta_{x}}{\mathrm{~d} t^{2}}>>\frac{\mathrm{d}^{2} X}{\mathrm{~d} t^{2}}$. Under these conditions, Eq 7 can be rewritten as

$$
\frac{\mathrm{d}^{2} \delta_{x}}{\mathrm{~d} \xi^{2}}=-\left(a_{x}-2 q_{x} \cos 2 \xi\right) X
$$


If there is no DC component so $a_{x}=0$ and assuming that $X$ is constant on the scale of $\delta_{x}, \mathrm{Eq} 11$ can be integrated twice to obtain $\delta_{x}$ in terms of $X$ as

$$
\delta_{x}=-\frac{1}{2} q_{x} X \cos 2 \xi
$$

Recalling that $2 \xi=\Omega t$, the $x$-coordinate can be written

$$
x=X-\frac{1}{2} q_{x} X \cos \Omega t
$$

Similarly,

$$
y=Y-\frac{1}{2} q_{\mathrm{y}} Y \cos \Omega t
$$

To a first approximation, the diminution of the twodimensional quadrupole potential in the fringing regions near the ends of the rods can be described by a function $f(z)$ so that the potential in the fringing regions can be written as

$$
\Phi_{\mathrm{FF}}=\Phi_{2 \mathrm{D}} f(z)
$$

and the axial component of the electric field due to the diminution of the two-dimensional quadrupole field is

$$
E_{z, \text { quad }}=-\Phi_{2 \mathrm{D}} \frac{\partial f(z)}{\partial z}
$$

When there is no DC component to the quadrupole potential, Eqs 4, 13, and 14 can be used in Eq 3 to write

$$
\begin{aligned}
\Phi_{2 \mathrm{D}}= & \frac{1}{r_{0}^{2}}(-V \cos \Omega t)\left[\left(X-\frac{1}{2} q_{x} X \cos \Omega t\right)^{2}-\left(Y^{2}\right.\right. \\
& \left.\left.-\frac{1}{2} q_{\mathrm{y}} Y \cos \Omega t\right)^{2}\right]
\end{aligned}
$$

Using Eq 17 in Eq 16

$$
\begin{aligned}
E_{z, \text { quad }}= & \frac{V}{r_{0}^{2}} \cos \Omega t\left[X^{2}\left(1-\frac{1}{2} q_{x} \cos \Omega t\right)^{2}-Y^{2}(1\right. \\
& \left.\left.-\frac{1}{2} q_{\mathrm{y}} \cos \Omega t\right)^{2}\right] \frac{\partial f(z)}{\partial z}
\end{aligned}
$$

In the case of an exit fringing-field, where $f(z)$ must be a decreasing function of $z$, the value of $E_{\mathrm{z}}$ averaged over one RF cycle $T_{\mathrm{RF}}$ can be obtained from

$$
\left\langle E_{\mathrm{z}, \text { quad }}\right\rangle_{\mathrm{RF}}=\frac{1}{T_{\mathrm{RF}}} \int_{t=0}^{T_{R F}} E_{\mathrm{z}, \text { quad }} \partial t
$$

Using Eq 18 in Eq 19, carrying out the integration and substituting for $V$ from $\mathrm{Eq} 9$

$$
\left\langle E_{z, \text { quad }}\right\rangle_{\mathrm{RF}}=\left|\frac{\partial f(z)}{\partial z}\right| \frac{m \Omega^{2}}{8 e}\left(q^{2}\left(X^{2}+Y^{2}\right)\right.
$$

where, because there is no DC component in the quadrupole potential, $q_{x}=-q_{y}=q$ has been used to simplify Eq 20. In most instances typical for MSAE, when thermal ions are excited resonantly by a dipolar auxiliary signal, secular amplitude increases appreciably only between the rods to which the auxiliary signal is applied and motion in the other coordinate direction would not contribute significantly to Eq 20.

Eq 20 shows that, as a consequence of the diminishing radial quadrupole potential in the fringing region, ions experience a net positive axial electric field, i.e., an electric field directed out of the linear ion trap. To a first approximation, this field is proportional to the square of the radial displacement, the square of the stability coordinate $q$, and the $\mathrm{m} / \mathrm{z}$ of the ion. It is noteworthy that this result is independent of the form of $f(z)$. However, to evaluate Eq 20, it remains to obtain $\partial f(z) / \partial z$.

Hunter and McIntosh [13] obtained an analytic expression for $f(z)$ by fitting an exponential function to a numerical solution of the Laplace equation. Adapting their expression to an exit fringing field, which terminates in the plane of the exit lens at $z=z_{1}$,

$$
f(z)=1-\exp \left(-a\left(z_{1}-z\right)-b\left(z_{1}-z^{2}\right)^{2}\right.
$$

The positive constants, $a$ and $b$, depend on the rod-lens separation and were tabulated for specific values in reference [13]. Differentiating Eq 21 with respect to $z$ one obtains

$$
\frac{\partial f}{\partial z}=-\left(a+2 b\left(z_{1}-z\right)\right)(1-f(z))
$$

which is negative for all $z<z_{1}$ as required.

\section{The Phase Relationship Between Ion Motion and the RF Potential}

The net positive axial field, which arises in consequence of the diminishing quadrupole field in the fringing region, can be understood, without specifying $f(z)$, by considering the relative phase of the micro-motion $\delta_{x}$ and that of the RF drive in a stability regime where the micro-motion is distinguished readily from higheramplitude secular motion, that is, where the approximation of Eq 10 is valid.

At any point of interest, the potential due to the RF drive is negative as often as it is positive, yet Eq 20 shows that there is a net positive axial force in the fringing region. Eq 12 shows that micro-motion in the $x-z$ plane is phase-synchronous with the potential applied to the $x$-rods, given by Eq 4. More generally, 

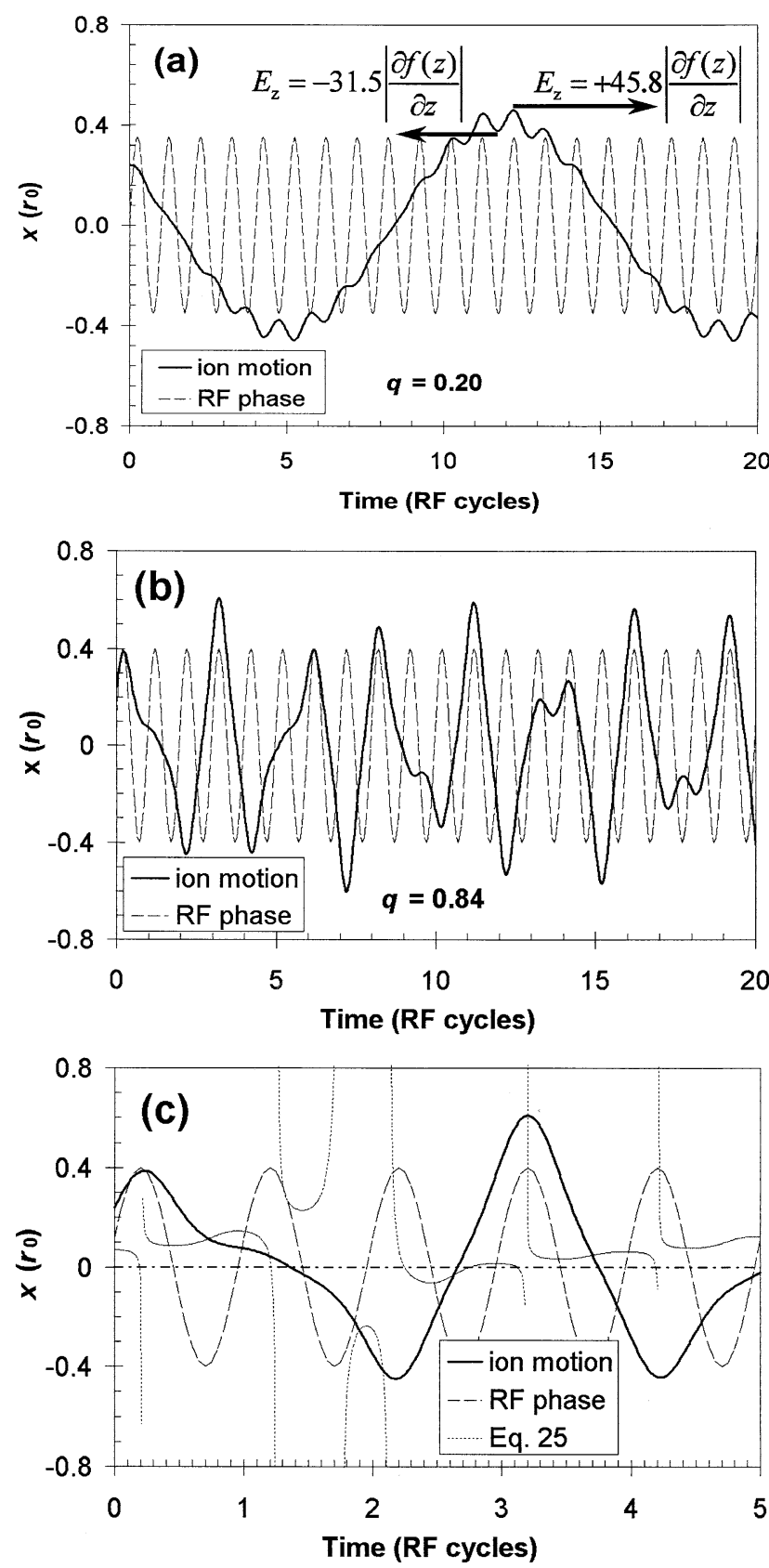

Figure 2. Maxima in the extrema of ions' motion and maxima in the local RF potential are always phase synchronous. Here, this phase relationship is demonstrated by superposing the phase of the RF voltage applied to the $x$-rods (dashed line) with the $x$-component of the trajectory of an ion of $\mathrm{m} / \mathrm{z}=609$ (solid line). (a) $(a, q)=(0,0.2)$ and $(\mathbf{b})(a, q)=(0,0.84)$. Using Eqs 3 and 4 in Eq 16 , the axial component of the electric field was evaluated at a minimum and a maximum of the ion's micromotion, as indicated in frame (a). Units of field strength are $\mathrm{V} / \mathrm{m}$. In frame (c) the left-hand-side of Eq 25 has been superposed with the first five RF cycles of frame $(\mathbf{b})$ to demonstrate that the phase relationship between the extrema of ions' radial motion and the local RF potential is independent of initial conditions.

maxima in ions' micro-motion and maxima in the local RF potential are in phase. This phase relationship has been illustrated in Figure 2a, where the RF potential applied to the $x$-rods has been superposed with the $x$-component of a simulated ion trajectory. The trajectory of Figure 2a was obtained with the RF amplitude adjusted to position $\mathrm{m} / \mathrm{z} 609$ at $q=0.2$, with $a=0$, in stability space.

In reference to $\mathrm{Eq} 18$, because $E_{z, \text { quad }}$ increases with the square of the radial displacement, its magnitude is always greatest when it is positive. Furthermore, as illustrated by Figure 2a, extrema of the micro-motion, whether positive or negative, occur during the positive half cycle of the RF. Consequently, the average of $E_{z, \text { quad }}$ over one RF cycle, $\left\langle E_{z, q u a d}\right\rangle_{\mathrm{RF}}$ of $\mathrm{Eq} 20$, is always positive. As an example, Eqs 3 and 4 were used in Eq 16 to evaluate $E_{z, \text { quad }}$ at a minimum and an adjacent maximum of the micro-motion of the simulated iontrajectory of Figure 2a. The magnitude of the negative axial field at the micro-motion minimum is only twothirds the magnitude of the positive axial field at the micro-motion maximum. Parenthetically, when Eq 18 was used to evaluate $E_{z, q u a d}$, nearly identical results were obtained. As a result, the average over one RF cycle is net positive, and in this example, Eq 20 evaluates to $+3.8\left|\frac{\partial f(z)}{\partial z}\right| \mathrm{V} / \mathrm{m}$.

At the stability coordinates of Figure 2a, the micromotion and secular motion are readily distinguished. However, the significance of higher-order terms in the complete solution to the Mathieu equation increases with increasing $q$ and this distinction diminishes above $q=0.45$. Even so, it can be demonstrated that maxima in stable ion trajectories remain phase synchronous with the RF potential when $q>0.45$. Figure $2 \mathrm{~b}$ demonstrates this condition for an ion of $m / z 609$ with $(a, q)=$ $(0,0.84)$. In this instance, initial conditions were $x=$ $0.24 \mathrm{r}_{0} \mathrm{~mm}, \dot{x}=1046 r_{0} / \mathrm{s}$ with an RF phase angle of $107^{\circ}$.

A more general approach to substantiate this claim can be taken by considering the complete solution to the Mathieu equation, Eq 7, given by the expression [14]

$$
\begin{aligned}
u(\xi)= & A \sum_{n=-\infty}^{\infty} C_{2 n} \cos (2 n+\beta) \xi \\
& +B \sum_{n=-\infty}^{\infty} C_{2 n} \sin (2 n+\beta) \xi
\end{aligned}
$$

where the $C_{2 n}$ coefficients are complicated functions of the Mathieu stability coordinates $a$ and $q$, and $A$ and $B$ are constants determined by initial conditions. Ion motion is the superposition of oscillations with frequencies $\omega_{n}$ given by

$$
\omega_{n}=(2 n+\beta) \frac{\Omega}{2}
$$

in which $n=0, \pm 1, \pm 2, \ldots$ In the stability regime where $a=0$ and $q<0.45$, the two dominant terms in Eq 
23 correspond to $n=0$ and $n=-1$ and the frequency of the micro-motion is the beat frequency of these two components. However, the significance of the other higher-order terms increases with increasing $q$ and above $q=0.45$, the increased amplitude of other frequencies overwhelms the two-component model. Even so, local radial maxima in ions' trajectories can be identified by setting the time-derivative of $\mathrm{Eq} 23$ to zero to obtain

$$
\frac{\sum_{n=-\infty}^{\infty} C_{2 n}(2 n+\beta) \cos (2 n+\beta) \xi}{\sum_{n=-\infty}^{\infty} C_{2 n}(2 n+\beta) \sin (2 n+\beta) \xi}-\frac{A}{B}=0
$$

To demonstrate, the first five RF cycles of the trajectory shown in Figure $2 b$ have been superposed with the left-hand-side of Eq 25 and the RF potential in Figure 2c, where the ordinates of the latter two have been scaled for clarity. From Figure 2c, it can be seen that solutions of Eq 25 occur at or near maxima of the RF potential. Furthermore, solutions occur most frequently at singularities of Eq 25, which are independent of the $A / B$ ratio, and hence of initial conditions.

\section{Examination of the Fields}

In this section, the theory developed above will be used to illustrate how axial fields in the fringing region influence ion trajectories. Central to understanding the impact of the diminishing quadrupole potential is the phase relationship between ion motion and the RF potential, discussed in the previous section. Relevant characteristics of the quadrupole and exit-lens fields will be discussed. Finally, the axial components of the quadrupole and exit-lens fields will be combined in suitable proportion to define a cone of reflection.

\section{Axial Field Due to Diminishing Quadrupole Potential}

To calculate numerical values for the average electric field strength, due to the diminishing quadrupole potential in the fringing region, $\left\langle E_{z, q u a d}\right\rangle_{R F}$, and the Hunter and McIntosh expression of $\mathrm{Eq} 22$ was used in $\mathrm{Eq} 20$. To obtain the data of Figure 3, $\left\langle E_{z, \text { quad }}\right\rangle_{\mathrm{RF}}$ was evaluated in the $x-z$ plane as a function of axial position for specific values of the secular amplitude $X$, specified in the legend in units of $r_{0}$. For these evaluations, the frequency of the RF drive was $1.0 \mathrm{MHz}$ and the RF amplitude was chosen to position $m / z 609$ at $q=0.45$. It is recognized generally that the Dehmelt approximation of $\mathrm{Eq} 8$, on which this evaluation of $\left\langle E_{z, q u a d}\right\rangle_{\mathrm{RF}}$ was based, decreases in accuracy with increasing $q$, and sees little application beyond $q=0.45$. Even more questionable is the use of the Hunter and McIntosh approxima-

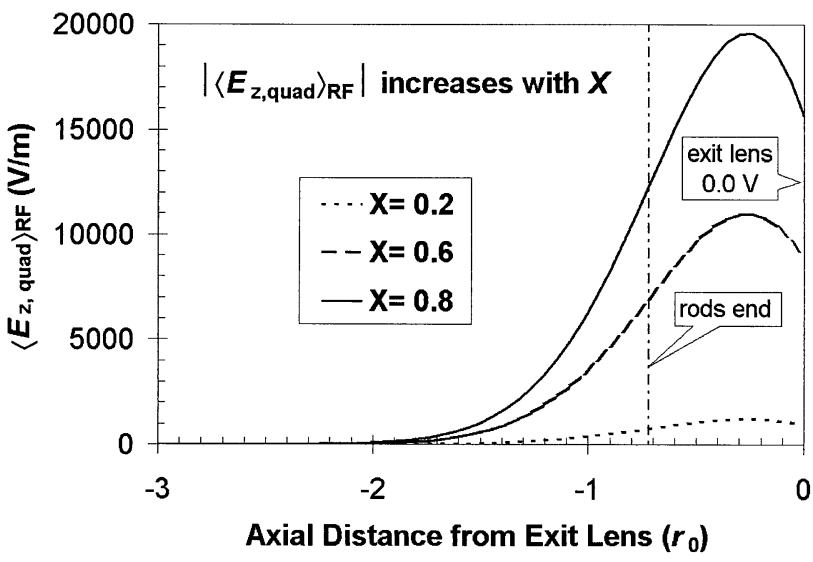

Figure 3. Analytic evaluation of $\left\langle E_{\mathrm{z}, \mathrm{quad}}\right\rangle_{\mathrm{RF}}$ in the $x$-z plane. With $Y=0$, Eq 20 was evaluated at $X=0.2,0.6$, and $0.8 r_{0}$ for an ion of $m / z=609$ at stability coordinates $(a, q)=(0,0.45)$. For any fixed $z$, $\left\langle E_{\mathrm{z}, \text { quad }}\right\rangle_{\mathrm{RF}}$ increases with the square of secular displacement, $X$.

tion at high radial amplitude. However, the purpose of this calculation was to identify salient features of specific contributions to fields in the fringing region that render MSAE practical, and these approximations suit this purpose well. The most significant features of Figure 3 are the strong increase in $\left\langle E_{z, q u a d}\right\rangle_{\mathrm{RF}}$ near the ends of the rods and, for any fixed $z$, that $\left\langle E_{z, \text { quad }}\right\rangle_{\mathrm{RF}}$ increases with the square of secular displacement, $X$. It is not surprising that these fields are strong. With $\mathrm{m} / \mathrm{z}$ 609 at $q=0.45$ and $r_{0}=4.17 \mathrm{~mm}$, the RF amplitude is about $487 \mathrm{~V}$ and that potential drops to zero over three millimetres between the ends of the rods and the exit lens. By comparison, the radial fields are even stronger. For example, under the same conditions, inside a rod array, where the fields are essentially two-dimensional, at $(x, y)=\left(0.8 r_{0}, 0\right)$ maxima in the radial field reach 1.9 $\times 10^{5} \mathrm{~V} / \mathrm{m}$.

\section{Axial Field Due to the Exit Lens Potential}

For effective MSAE, the positive electric field described by Eq 20 must be balanced, in suitable proportion, by a negative electric field, which decreases in strength with increasing secular displacement; otherwise, all ions would be ejected axially with no mass discrimination. This condition can be achieved simply by applying a static positive DC potential to the exit lens as $U_{\text {lens. }}$. Since no analytic expression exists for this contribution, specific values for $E_{z, \text { lens' }}$ the axial component of the electric field arising from the exit lens potential, were obtained by differentiating a numerical solution to the Laplace equation. The contribution of $E_{z, \text { lens }}$ to the total electric in the $x$-z plane, for a lens potential of $U_{\text {lens }}=2.5$ $\mathrm{V}$, has been plotted in Figure 4 as a function of axial position. As in Figure 3, specific values of the secular amplitude $X$, are specified in units of $r_{0}$. In contrast to $\left\langle E_{z, q u a d}\right\rangle_{R F}$ of Figure 3, $\left|E_{z, \text { lens }}\right|$ decreases with increasing $X$ for all $z<-0.9 r_{0}$ as required. 


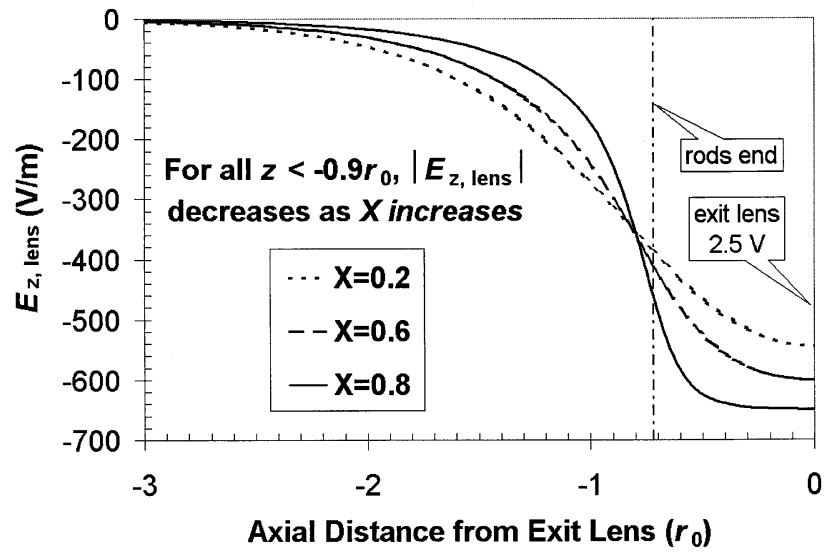

Figure 4. Numerical evaluation of $E_{z, \text { lens }}$ in the $x-z$ plane. Fields were obtained by solving the Laplace equation numerically and differentiating the result. $E_{z, \text { lens }}$ was evaluated at $X=0.2,0.6$, and $0.8 r_{0}$ for an ion of $\mathrm{m} / \mathrm{z}=609$ at stability coordinates $(a, q)=(0$, $0.45)$. For all fixed $z<-0.9 r_{0},\left|E_{z, \text { lens }}\right|$ decreases as the secular displacement $X$ increases.

\section{Cone of Reflection}

The two contributions to the axial component of the electric field, $\left\langle E_{z, \text { quad }}\right\rangle_{\mathrm{RF}}$ of Figure 3 and $E_{z \text {,lens }}$ of Figure 4 , were summed to obtain the total axial field experienced by an ion, averaged over one RF cycle $\left\langle E_{z}\right\rangle_{\mathrm{RF}}$. The result has been plotted as a function of axial position in Figure 5a using an expanded vertical scale so that the zero-crossings (circled) are apparent. For most values of $X$, there is a value of $z$ for which $\left\langle E_{z}\right\rangle_{\mathrm{RF}}=0$. The locations of these zero-crossings in the $x-z$ plane are shown in Figure $5 b$ with solid circles. These points define a surface on which the net axial force acting on an ion, over one RF cycle, is zero. In recognition of its shape, this surface has been dubbed the cone of reflection. Ions inside (to the left) the cone of reflection experience a negative axial force away from the exit lens, while those outside (to the right) experience a net positive force toward the exit lens. When the exit lens potential is chosen judiciously, ions with sufficient radial amplitude are able to penetrate this surface, and be ejected axially, while all others are reflected.

\section{Mapping the Cone of Reflection Numerically}

In the previous section, a cone of reflection was obtained using a combination of analytic theory for the quadrupole contribution and numerical methods to obtain the contribution from the exit-lens potential. To test the accuracy and range of applicability of the analytic theory, it was undertaken to obtain cones of reflection using numerical methods only. However, in contrast to the static field arising from a fixed potential applied to the exit lens, the axial field strength due to the quadrupole potential is time dependent. Therefore, simply calculating the axial field strength at specific points was not an adequate treatment. Rather, it was

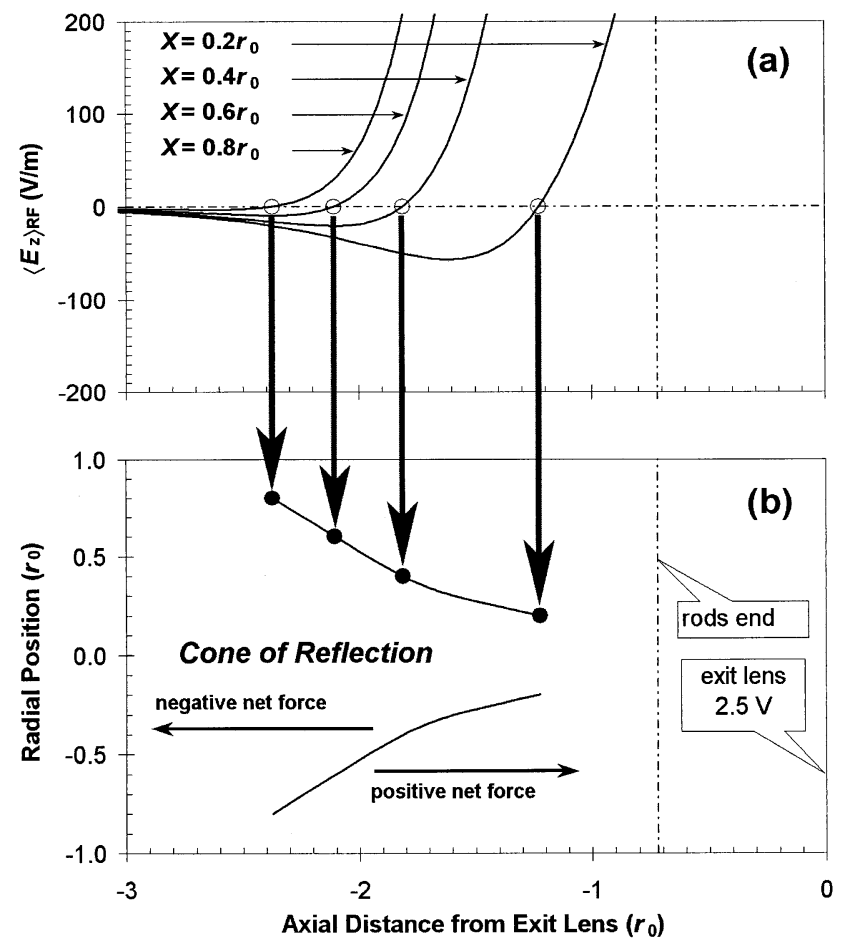

Figure 5. Mapping the cone of reflection. Frame (a) shows the superposition of $\left\langle E_{z, q u a d}\right\rangle_{\mathrm{RF}}$ of Figure 3 with $E_{z, \text { lens }}$ of Figure 4 to obtain the total axial field experienced by an ion of $m / z=609$ at stability coordinates $(a, q)=(0,0.45)$ averaged over 1 RF cycle $\left\langle E_{\mathrm{z}}\right\rangle_{\mathrm{RF}}$. In frame (a) the ordinate has been expanded to illustrate the zero-crossings (circled). The locations of these zero-crossings in the $x-z$ plane, shown as solid circles in frame $(\mathbf{b})$, define the cone of reflection.

necessary to develop a scheme wherein ions were used probes to measure $\left\langle E_{\mathrm{z}}\right\rangle_{\mathrm{RF}}$, the net effective axial field experienced by an ion, averaged over one RF cycle. Finally, trajectory simulations of ions undergoing massselective axial ejection were used to identify loci of points in the fringing region at which axial ejection was assured and these loci were compared to numerically generated cones of reflection, obtained under similar circumstances.

Two techniques, in which ions were used as probes, were developed to map cones of reflection. In the first, with their axial coordinates held constant, thermal ions were excited radially. With the second, ions with random radial amplitudes were moved toward the exit lens with constant axial speed. The second technique was developed for situations for which the first was poorly suited. Both techniques returned mutually consistent results, and in many instances, the results of both were combined to obtain a complete mapping. In all instances where trajectory calculations were used to obtain cones of reflection, the simulations were carried out in collision-free environments. Cones of reflection, which were obtained when collisions with a buffer gas were enabled, differed little in position from their collision-free counterparts, but were defined less sharply. Each of the three techniques used to obtain 
cones of reflection numerically are described in turn below.

\section{Technique of Constant Axial Coordinate}

In this method, the axial coordinates of initially thermalized ions were fixed at random values, which spanned the fringing region and the ions were excited resonantly using an auxiliary two-dimensional dipolar signal. The only purpose of the auxiliary signal was to increase gradually the radial amplitudes of the ions. Typically, the amplitude of the auxiliary signal was less than one volt. During the excitation, with the amplitude of the quadrupole RF drive and the exit lens potential held constant, ions were allowed to respond radially, but their axial coordinates were held fixed.

Initially, while their radial amplitudes were relatively low, ions felt a net negative axial force (away from the exit lens). With increasing radial amplitude, the net axial force became increasingly positive. Upon gaining sufficient radial amplitude that the net axial force acting on an ion, averaged over one RF cycle, became positive, its maximum radial displacement was plotted against its axial position to define a single point on the cone of reflection. Many such points, typically one thousand, were accumulated to define a single cone.

For this scheme to work, it was necessary to adjust the frequency of the auxiliary signal to compensate for the diminishing RF level in the fringing region. For the simulations, which used Eq 2, the quadrupolar field was pure and Eq 21 specified its diminution. In consequence, the appropriate auxiliary frequency for each axial position could be calculated from the continuedfraction expression for $\beta$ in terms of Mathieu stability coordinates [14]. Stable, periodic solutions to the Mathieu equation exist only for non-integral values of the dimensionless parameter $\beta$ and the fundamental angular frequency of ions with stable trajectories is given by $\omega=0.5 \beta \Omega$, where $\Omega$ is the angular frequency of the RF drive [11]. However, for those cases where the quadrupole field was determined numerically, Eq 1, this technique was no longer effective. Instead, the simulator was operated in parametric mode to identify the frequency to which ions responded most strongly at millimetre intervals of the axial coordinate. These values were fit to equations similar to Eq 21, with adjustable coefficients to obtain an analytic expression from which appropriate frequencies for the auxiliary dipolar signal could be calculated at runtime. It is interesting that, due to the dramatic increase in the significance of higher-order terms in the essentially quadrupole potential, within about $0.7 r_{0}$ of the ends of the rods, no frequency could be found at which an auxiliary signal of any reasonable amplitude (less than five volts) was capable of driving ions to the rods. Specifically, as higher-order terms contribute proportionately more to the potential, the radial field becomes increasingly nonlinear and secular frequency becomes increasingly
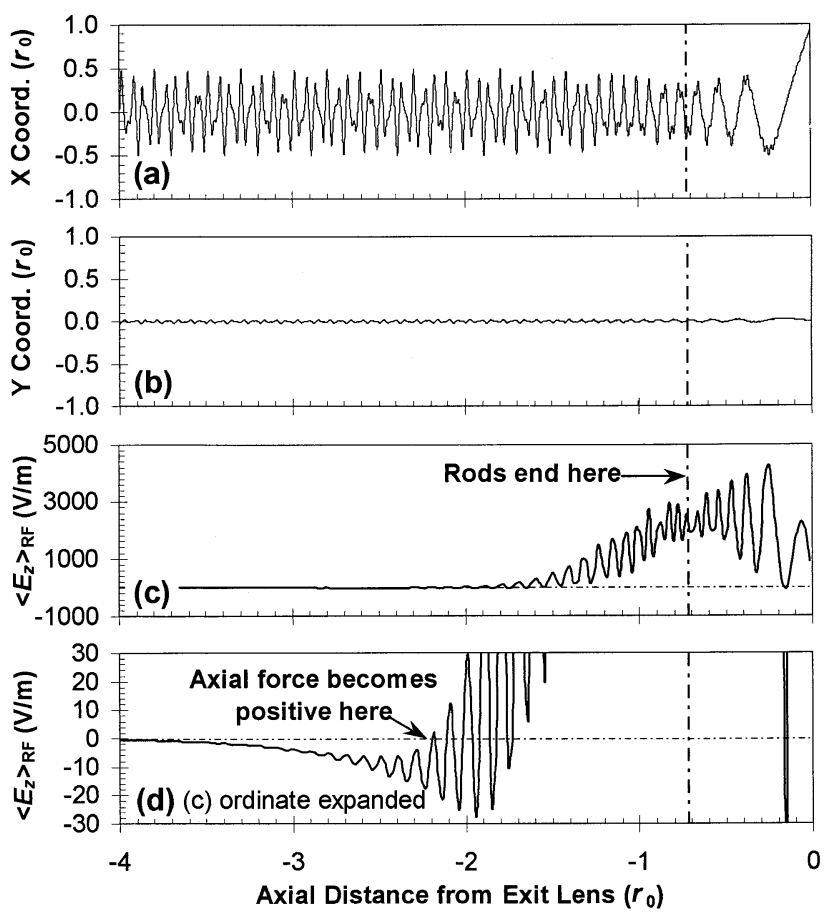

Figure 6. Obtaining a point on the cone of reflection by the technique of constant axial speed. Frames (a) and (b) show motion in the two radial coordinates, $x$ and $y$, respectively, plotted as a function of axial position. Frame (c) shows $\left\langle E_{\mathrm{z}}\right\rangle$, the net axial electric field strength experienced by an ion, averaged over one RF cycle. Frame (d) shows $\left\langle E_{\mathrm{z}}\right\rangle$ with the ordinate expanded to show that point where $\left\langle E_{z}\right\rangle$ first becomes positive.

amplitude-dependent. In consequence, ions go off-resonance as their amplitude increases and suffer cycles of excitation and de-excitation rather than sustained amplitude growth.

\section{Technique of Constant Axial Speed}

Due to the non-linearity of fields in the fringing region, it was necessary to map some cones, or at least some portions of some cones, by a different method. Specifically, in a two-dimensional environment, dipolar resonant excitation was used to excite thermal ions to random radial amplitudes between zero and $r_{0}$. This ion list was saved and used as input to a calculation in which ions were allowed to move freely in the radial directions as before, but were constrained to move axially through the fringing region from $z=-4.8 r_{0}$ toward the exit lens at the constant speed of $100 \pm 2.5$ $\mathrm{m} / \mathrm{s}$ (about $0.1 \mathrm{~mm}$ per RF cycle). As before, the axial position, combined with the maximum radial position of the ion during the first RF cycle over which the average axial electric field became positive, defined a single point on the cone of reflection.

This technique of using constant axial speed has been illustrated in Figure 6 where specific features of an ion's trajectory $(m / z 609, q=0.84)$ have been plotted as a function of its axial position as it was moved through the fringing region from $z=-4.8 r_{0}$ toward the exit lens. 
The $x$ and $y$ coordinates of the ion, output at regular increments 20 times each RF cycle in Figure 6a and b, show that the radial amplitude of the ion, about $0.5 r_{0}$, is confined primarily to the $x-z$ plane and remains relatively constant until the axial force becomes positive. Figure $6 \mathrm{c}$ which shows the axial field strength, averaged over one RF cycle, experienced by the ion as it was moved from $z=-4 r_{0}$ toward the exit lens at $z=0$. The expanded ordinate of the second frame, Figure $6 \mathrm{~d}$ shows clearly the point at which the net axial force, averaged over one RF cycle, became positive, yielding a point on the cone of reflection at $x=-0.5 r_{0}$ and $z=$ $-2.2 r_{0}$. In addition, Figure $6 \mathrm{c}$ illustrates a feature of the fields in the fringing region that is highly significant for MSAE. Specifically, the strength of the axial field is very weak to the left of the cone of reflection, but increases dramatically to the right. The consequence is that once an ion penetrates the cone it is ejected promptly, typically within a few RF cycles.

\section{Obtaining Cones of Reflection from Trajectory Data}

In the two techniques described above, ions were used as probes to identify coordinates where the net effective axial force experienced by ions, averaged over one RF cycle, was zero. Cones of reflection, obtained by these methods, do not correspond necessarily to those points in ions' trajectories at which axial ejection becomes assured. Indeed, in simulations in which ions were released from their axial constraints and allowed to respond freely to the axial fields when the net axial force averaged over one RF cycle first became positive, were reflected promptly without exception. Therefore, it is of interest to compare the loci of points obtained by these methods to those points in the trajectories of ions at which axial ejection became assured.

To obtain such data, it was necessary to simulate conditions suitable for MSAE and examine closely the trajectories of those ions, which were ejected axially. To this end, a population of singly charged positive ions of mass $609 \mathrm{Da}$ were allowed to thermalize, through collisions with a nitrogen buffer gas, under conditions that precede MSAE under normal experimental conditions. Subsequently, the RF amplitude was ramped at a rate corresponding to $1000 \mathrm{Da} / \mathrm{s}$ to bring ions into resonance with a dipolar auxiliary signal of frequency $380 \mathrm{kHz}$, corresponding to $q=0.84$ for an RF drive frequency of $1.0 \mathrm{MHz}$. As they came into resonance with the auxiliary signal, ions were either ejected axially or lost on the rods. To improve the ratio of ions, which were ejected axially, to those lost on the rods, the cell was made as short as possible by eliminating the middle section of the rod array where the fields are two-dimensional. As a result, the cell consisted of an input fringing field followed immediately by an exit fringing field, yielding a cell of overall length $40 \mathrm{~mm}$. In addition, efficiency was improved further by allowing
MSAE to occur at both ends. Figure 7 shows a trajectory, which is characteristic of an ion that is ejected axially in a mass-selective way. With the exception of frames $\mathrm{d}$ and e, the ordinates of Figure 7 indicate averages of twenty evaluations, made at regular intervals in time, each RF cycle. Specifically, Figure 7a, b, and $\mathrm{c}$ show the $x, y$, and $z$ components of the ion's trajectory. Figure $7 \mathrm{~d}$ shows the maximum radial excursion in the ion's radial amplitude, which occurred during each RF cycle and frame e shows, on an expanded abscissa, the final $104 \mu \mathrm{s}$ of the frame $\mathrm{d}$ data. Figures $7 \mathrm{f}, \mathrm{g}$, and $\mathrm{h}$ show the $x, y$, and $z$ components of the ion's velocity expressed in units of energy $(\mathrm{eV})$. Finally, Figure 7i shows the axial component of the ion's velocity on a scale where the final point of inflection is clearly visible and frame $j$ shows, on an expanded abscissa, the final $104 \mu \mathrm{s}$ of the frame i data. The last point of inflection (true minimum) in the axial component of the ion's trajectory, indicated with an arrow in Figure $7 \mathrm{i}$ and with a triangle in Figure 7j, was used to identify the axial point of no return in the process of MSAE, and the axial coordinate of a point on the cone of reflection.

The obvious choice for the radial coordinate of this point on the cone of reflection would be the point from Figure 7e corresponding in time to the point of inflection in Figure 7j. Both of these points are marked with triangles. However, careful examination of a number of trajectories showed that the points of inflection occurred randomly relative to the phase of the radial maxima illustrated in Figure 7e. It was found that the most consistent representation of cones of reflection obtained by choosing, for the radial coordinate, the maximum in the ion's trajectory, which occurred over a few RF cycles ( 3 or 4 ) immediately preceding the point of inflection, rather than the specific radial maximum that occurred on the same RF cycle as the point of inflection. Arrows in both in Figure $7 d$ and e indicate this point. Invariably, this point was very close to the overall radial maxima in ions' trajectories.

To accumulate a sufficient number of points to define a cone of reflection, it was necessary to process a large number of ions, typically one thousand. As a result it was essential to define an automated algorithm for identifying these points in files of trajectory data. Explicitly, the point of inflection was determined by searching backwards through the axial-energy data until the first minimum was identified. False minima were avoided by requiring that the preceding eight values were larger than the current candidate, before terminating the search. As described above, a corresponding radial coordinate was chosen by identifying the maximum in the ion's radial amplitude, over a few preceding RF cycles.

\section{Results from Simulation and Experiment}

For the results reported here, a variety of techniques were used to examine the impact, both on the cone of 

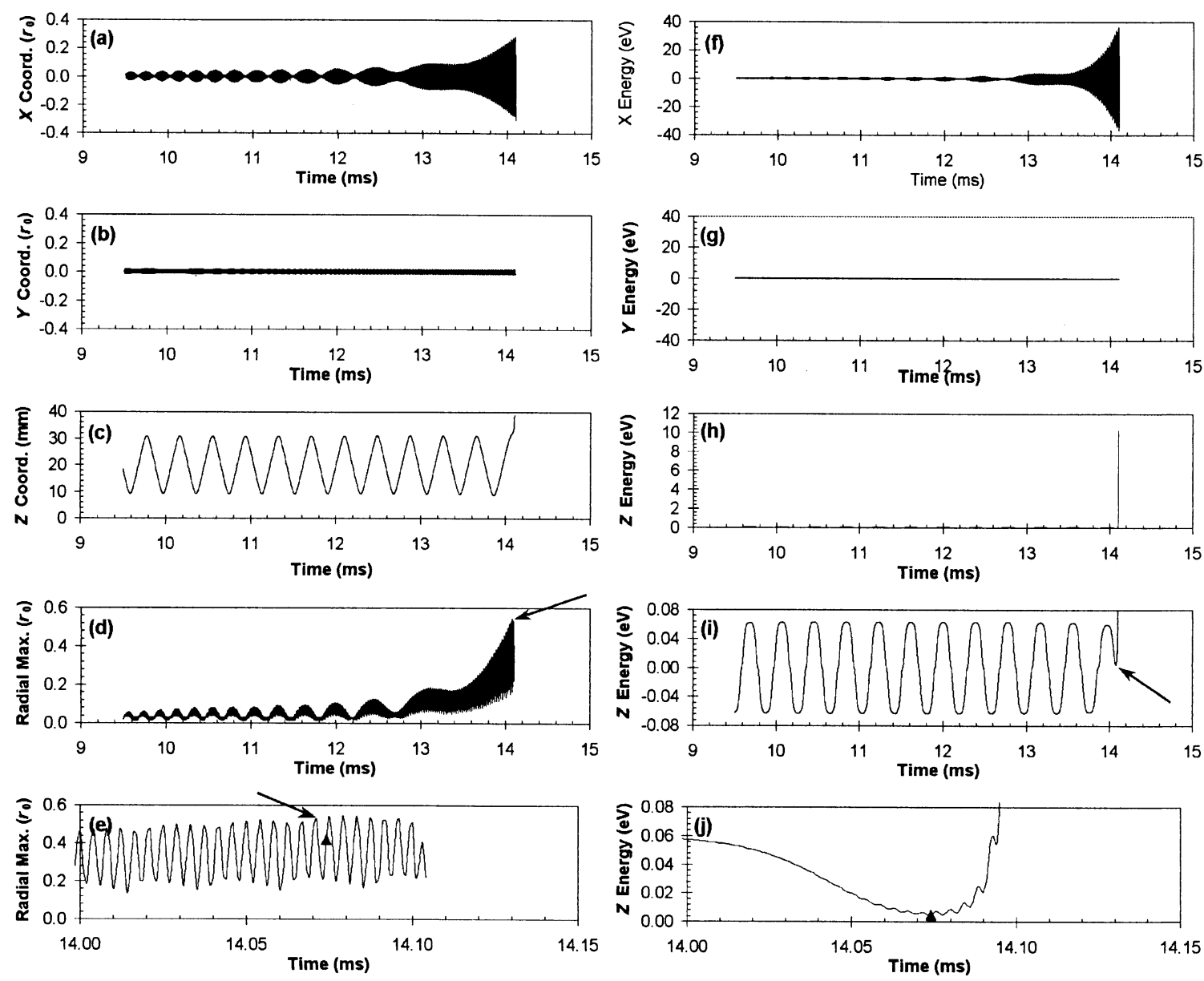

Figure 7. A trajectory characteristic of an ion that is ejected axially in a mass-selective way. With the exception of frames $(\mathbf{d})$ and $(\mathbf{e})$, the ordinates indicate averages of twenty evaluations, made at regular intervals in time, each RF cycle. Frames (a), (b), and (c) show the $x, y$, and z components of the ion's trajectory. Frame (d) shows the maximum radial excursion in the ion's radial amplitude, which occurred during each RF cycle and frame (e) shows, on an expanded abscissa, the final $104 \mu \mathrm{s}$ of the frame (d) data. Frames (f), (g), and (h) show the $\mathrm{x}, \mathrm{y}$, and $\mathrm{z}$ components of the ion's velocity expressed in units of energy $(\mathrm{eV})$. Frame (i) shows the axial component of the ion's velocity on an ordinate scale where the final point of inflection is clearly visible and frame (j) shows, on an expanded abscissa, the final $104 \mu \mathrm{s}$ of the frame (i) data. The last point of inflection (true minimum) in the axial component of the ion's trajectory, indicated with an arrow in frame (i) and with a triangle in frame $(\mathbf{j})$, was used to identify the axial point of no return in the process of MSAE. The corresponding point in time is indicated by a triangle in frame (e). The arrow in frame (e) indicates the maximum in the ion's trajectory over a few RF cycles (3 or 4) immediately preceeding the point of inflection.

reflection and on mass-spectral data, of the Mathieu stability parameter $q$, the exit lens potential, and the amplitude of the auxiliary signal used to excite ions radially. In addition, cones of reflection obtained by different methods are compared to test the validity both of the theory and of the numerical techniques used to generate them. Finally, simulated mass-intensity signals, and corresponding cones of reflection, are examined to evaluate the relationship between these two, and determine if features of the cone of reflection can offer any insights into the nature of mass-spectra.

\section{The Impact of Mathieu Stability Parameter $q$}

The value of the Mathieu stability parameter $q$ at which MSAE occurs is determined by the frequency of the auxiliary signal used to excite resonantly the radial motion of ions. To examine the impact of changes in ejection- $q$ on the cone of reflection, and to explore the suitability, in this application, of the Dehmelt approximation, Eq 10, at higher values of $q$, cones of reflection were generated both analytically and by using ions as probes as described above.

Using $m / z 609$ with a constant exit-lens potential $V_{\text {lens }}$ $=2 \mathrm{~V}$, cones of reflection were mapped analytically, by the technique illustrated in Figure 5, for ejection- $q$ values of $0.20,0.45$, and 0.84 . These are shown in Figure 8 with dotted, dashed and solid lines, respectively. For comparison, cones of reflection for these values of ejection- $q$ were generated with the simulator by using ions as probes as described above. These are shown with exes, circles and squares, respectively, in Figure 8. 


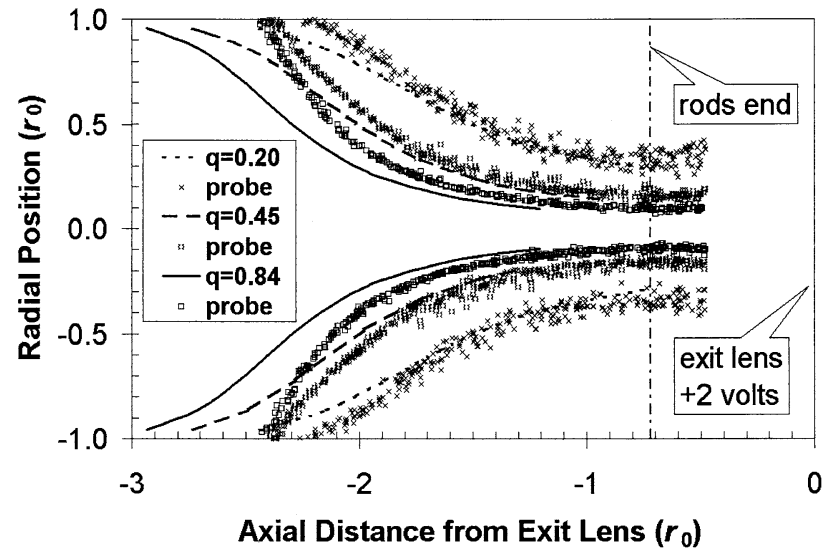

Figure 8. Cones of reflection obtained for $m / z 609$ with a constant exit-lens potential of $2 \mathrm{~V}$. Results obtained analytically, by the technique illustrated in Figure 5, for ejection- $q$ values of 0.20, 0.45, and 0.84 are shown with dotted, dashed, and solid lines, respectively. Cones of reflection for the same ejection- $q$ values, mapped numerically by using ions as probes, are shown with exes, circles and squares, respectively.

In these simulations, ion trajectories were calculated by integrating Eq 2 with $\Phi_{\mathrm{FF}}$ specified by Eq 15. Recall that Eq 15 simply describes a two-dimensional quadrupole field, which diminishes in the fringing region according to the approximation of Hunter and McIntosh, Eq 21. Although numerically calculated fields would be expected to return a more accurate result, Eq 15 was used as a basis for the analytic results and provides the most direct test of the theory based on the Dehmelt approximation.

It is clear from Figure 8 that the differences between cones obtained analytically (solid lines) and through simulation, using ions as probes, increase both with the Mathieu stability parameter $q$ and with radial amplitude. Both techniques were based on the same fields, but the quadrupole term was treated differently in the two cases. The analytic results relied upon the Dehmelt approximation, embodied in Eq 20 to obtain $\left\langle E_{z, \text { quad }}\right\rangle_{\mathrm{RF}}$. In the simulations, which used ions as probes to map cones of reflection for Figure $8, \Delta\left[\Phi_{2 \mathrm{D}} f(z)\right]$ was evaluated by using $\mathrm{Eq} 3$ in $\mathrm{Eq} 15$ and the result was integrated numerically to obtain ion trajectories. In other words, the differences between the analytic and numerical results presented in Figure 8 are due primarily to the use by the analytic technique of the Dehmelt approximation, whose accuracy is well known to diminish with increasing $q$.

Additional inaccuracy in the results of Figure 8 was introduced by the Hunter and McIntosh expression for $f(z)$ whose reliability diminishes rapidly with increasing radial amplitude. However, this approximation was common to both treatments and should not have contributed to the discrepancy in the results. Despite the inaccuracies inherent in the Dehmelt and Hunter and McIntosh approximations, the expression for $\left\langle E_{z, q u a d}\right\rangle_{\mathrm{RF}}$ given by Eq 20 does provide some useful insights into the process of MSAE.

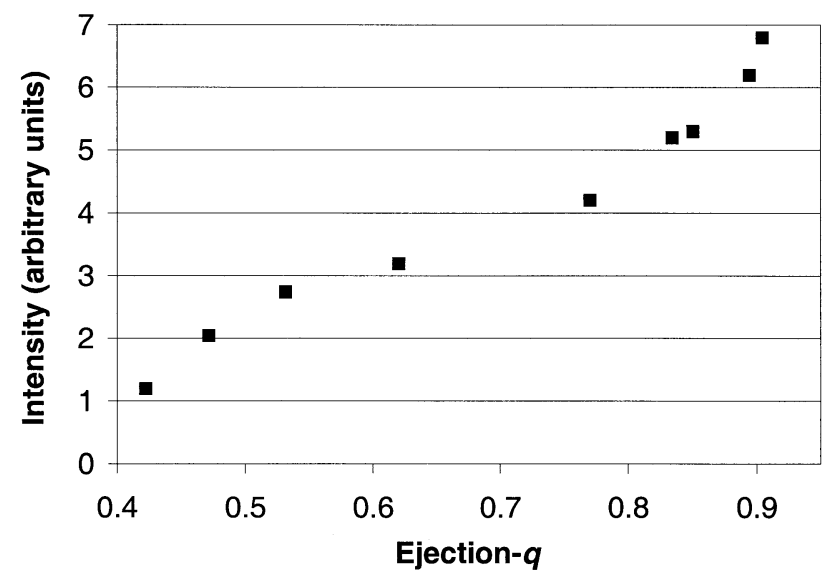

Figure 9. The integrated ion signal for MSAE of $\mathrm{m} / \mathrm{z} 609$ measured experimentally as a function of ejection- $q$ for an exit-lens potential of $2 \mathrm{~V}$.

For example, according to Eq 20, the strength of $\left\langle E_{z, \text { quad }}\right\rangle_{\mathrm{RF}}$ increases with the square of $q$ and this relationship is reflected in the cones of reflection in Figure 8. Clearly, the cone of reflection corresponding to $q=0.2$ would be the least conducive to MSAE because ions would need to advance the furthest into the fringing region against the repelling force of the exit-lens potential and in addition would require very high radial amplitude to penetrate the cone. Although this situation could be improved by reducing the potential on the exit lens, experimentally, the sensitivity and resolution of MSAE improve with increasing $q$, reaching broad maxima up to $q=0.904$. This is demonstrated in the experimental results presented in Figure 9 in which the integrated ion signal for MSAE of $\mathrm{m} / \mathrm{z}$ 609 was measured as a function of ejection- $q$ with an exit-lens potential of 2 volts.

\section{Impact of the Potential on the Exit Lens}

For MSAE to be effective, the exit lens potential must be chosen judiciously. When it is reduced below optimum, resolution is degraded and, in more extreme cases, mass discrimination is lost. As the exit-lens potential is increased above optimum, sensitivity suffers and ultimately ion current is reduced to zero. To examine the impact of this parameter on the cone of reflection, the ion-probe method was used to map cones of reflection for exit-lens potentials of 2, 4, and $8 \mathrm{~V}$. As before, the RF drive frequency was $1.0 \mathrm{MHz}$ and in this case the RF amplitude adjusted to position $\mathrm{m} / \mathrm{z} 609$ at $q=0.45$. These results are presented in Figure 10.

Cones of reflection obtained by using Eq 15 to specify $\Phi_{\mathrm{FF}}$ in Eq 2 are shown in Figure 10a. These were generated using the same technique and equations described above, with reference to the numerically generated cones of Figure 8. In fact, cone in Figure 10a for which the exit-lens potential is $2 \mathrm{~V}$ is identical to the numerically generated cone in Figure 8 for $q=0.45$. As mentioned above, the accuracy of the Hunter and 

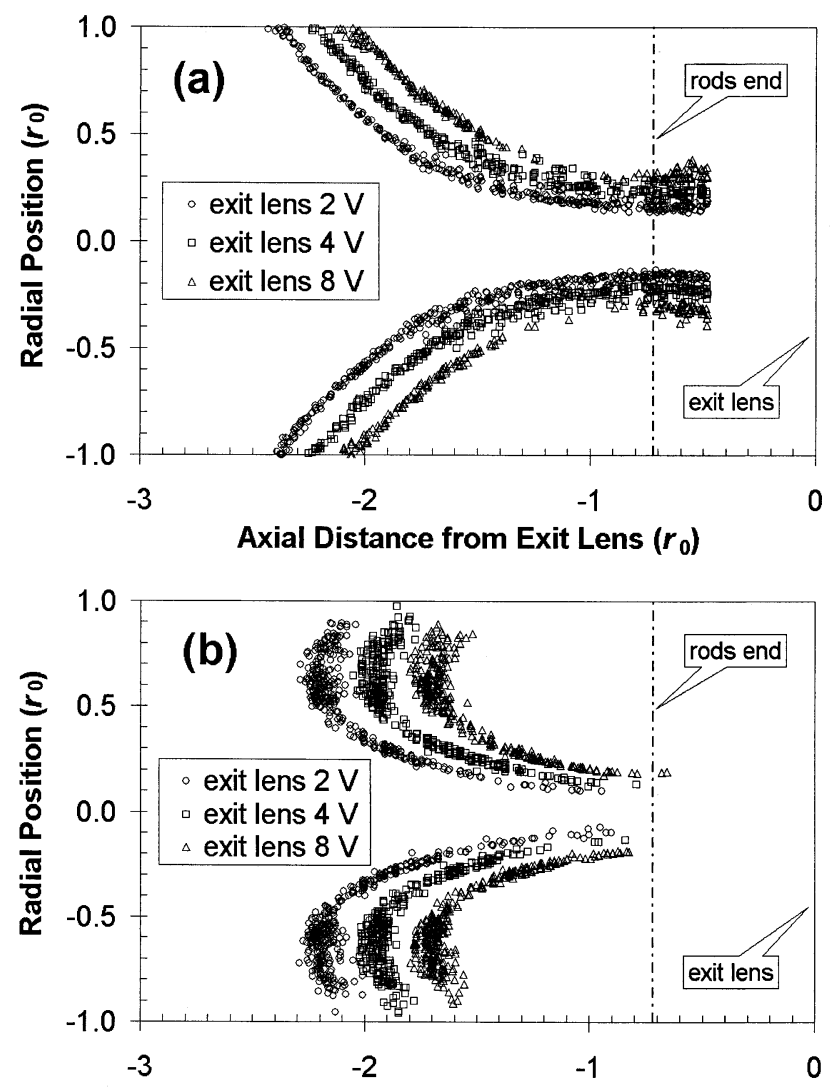

Axial Distance from Exit Lens $\left(r_{0}\right)$

Figure 10. Cones of reflection obtained for $m / z=609$ at stability coordinates $(a, q)=(0,0.45)$ for exit lens potentials of 2,4 , and $8 \mathrm{~V}$ by using ions as probes: (a) by using Eq 15 to specify $\Phi_{F F}$ in Eq 2 and (b) by using a full numerical solution to the Laplace equation for quad in Eq 1.

McIntosh expression for $f(z)$ decreases with increasing radial amplitude. Moreover, the approximation of Eq 15 precludes higher-order terms in $x$ and $y$, whose significance increases dramatically near the ends of the rods. Therefore, to determine the impact of these inaccuracies, cones of reflection, which were mapped using a full numerical solution to the Laplace equation for $\vec{E}_{\text {quad }}$ in Eq 1, are shown in Figure 10b. From these results, the limitations of the Eq 15 approximation are apparent.

In both frames of Figure 10, the cones of reflection remain similar in shape, but move closer to the exit lens in response to an increasing exit-lens potential. This behavior is consistent with the experimental observations cited at the beginning of this section. Those claims can be restated with explicit reference to the cone of reflection. Specifically, when the exit-lens potential is too low, the cone of reflection is further from the exit lens inside the rod array. As a result, the cone can be penetrated by ions with only modest radial amplitude or, in some cases, even by thermal ions with higher axial energy. As the cone is moved toward the ends of the rods, in response to increasing potential on the exit lens, ions require increasingly higher radial amplitude and/or axial energy to penetrate the cone and be detected.

\section{Cones Obtained from Trajectory Data}

Up to this point, cones of reflection have been mapped by three different methods of direct calculation. Specifically, these methods, listed in order of increasing accuracy, were: analytic, based on the Dehmelt and Hunter and McIntosh approximations; numeric, based on the Hunter and McIntosh approximation only; and finally, full numeric, based on complete numerical solutions to the Laplace equation in the fringing region. In all of these cases, cones were mapped by identifying loci of points in the fringing region at which the net axial force experienced by ions, averaged over one RF cycle, was zero; that is, $\left\langle E_{\mathrm{z}}\right\rangle_{\mathrm{RF}}=0$. It remains to compare cones of reflection, which conform to this definition, to those points in ions' trajectories at which axial ejection becomes assured.

The technique used to obtain cones of reflection from the trajectories of ions that experienced MSAE, and specific conditions of those simulations, were discussed in some detail above. The points of inflection in trajectory data obtained from those simulations, which identify the point-of-no-return for ions undergoing MSAE under similar conditions, are shown with large circles in Figure 11. In Figure 11, frames a, b, and c correspond to exit-lens potentials of $1.0,2.0$, and $4.0 \mathrm{~V}$, respectively. In all cases, the amplitude of the dipolar auxiliary signal used for resonant excitation was $0.2 \mathrm{~V}_{0-p}$.

For comparison, cones of reflection obtained by the most accurate of the numerical methods, which used ions as probes to identify $\left\langle E_{\mathrm{z}}\right\rangle_{\mathrm{RF}}=0$, are shown in Figure 11 with letter $x$. These data were obtained using singly charged ions of mass $609 \mathrm{Da}$ positioned in stability space at $q=0.84$. In addition, an analytic result based on the Dehmelt and Hunter and McIntosh approximations and obtained by the technique illustrated in Figure 5, is shown with a solid line in Figure 11b.

The degree of similarity between the two representations in Figure 11 is most encouraging and the differences are consistent with previous discussion. Recall that the exes identify coordinates where the net axial force averaged over one RF cycle first became greater than or equal to zero. Since positive axial force increases with radial displacement, as described by Eq 20, it would be expected that this condition would be met near a maximum of the secular motion, and that the average radial amplitude of ion during the following RF cycle would be lower, and that the net average axial force experienced by an ion on that next RF cycle would be negative. In fact, when simulations, in which ions were used as probes to map the cone of reflection, were allowed to proceed with axial response enabled, after the condition $\left\langle E_{z}\right\rangle_{\mathrm{RF}} \geq 0$ had been met, ions were reflected promptly and without exception. Therefore, one would expect that the point in an ion's trajectory at which axial ejection was assured would lie outside of the cone defined by $\left\langle E_{z}\right\rangle_{\mathrm{RF}}=0$. However, as illustrated 

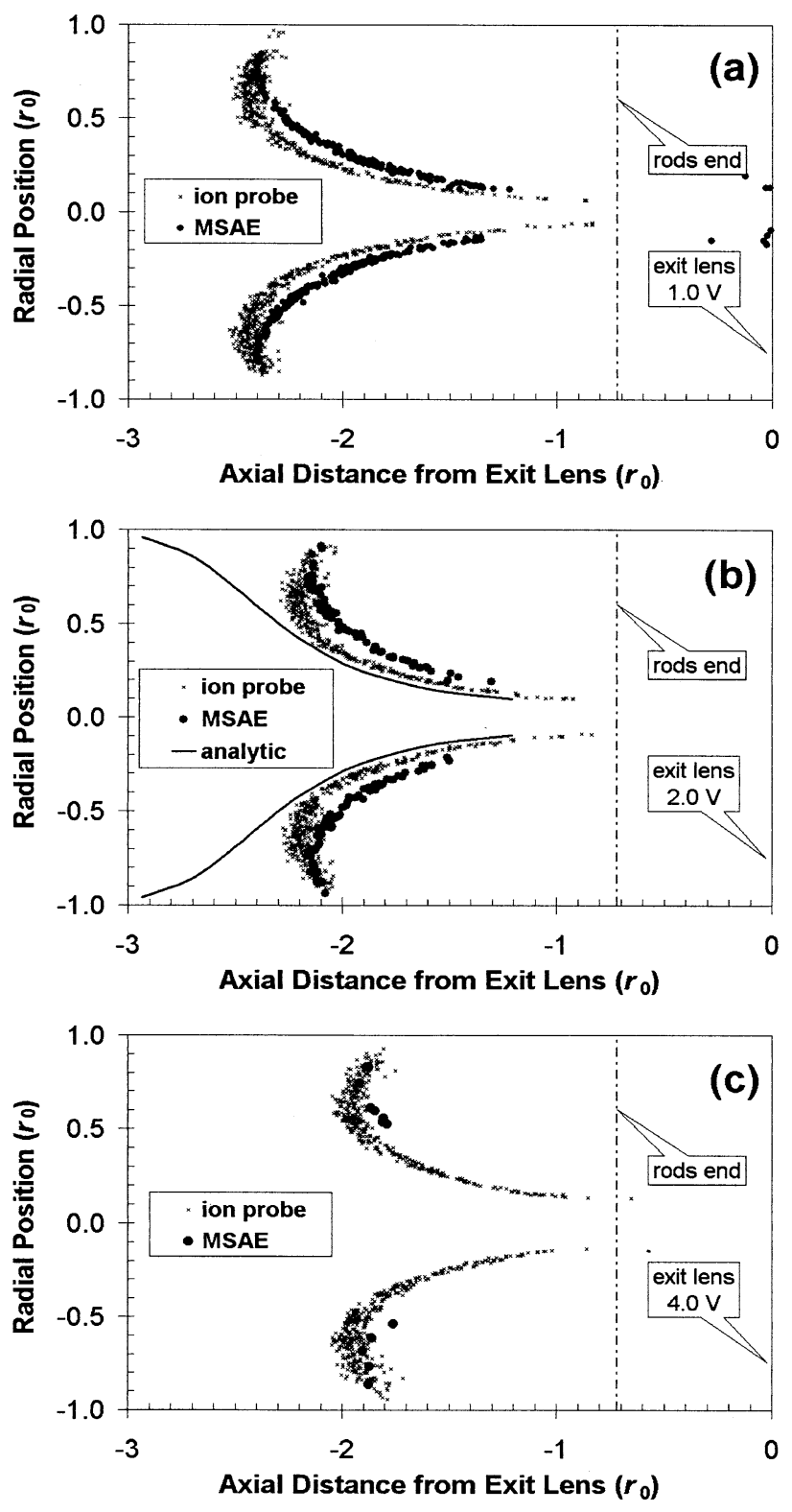

Figure 11. Cones of reflection obtained for $m / z 609$ ions positioned in stability space at $q=0.84$ with exit-lens potentials of 1.0, 2.0 , and $4.0 \mathrm{~V}$ in frames (a), (b), and (c), respectively. Exes identify cones of reflection, obtained from direct numerical calculations employing a full numerical solution to the Laplace equation using ions as probes. Approximately 1000 ion-probes were used for each exit-lens potential. The points of inflection in trajectory data, which identify the point-of-no-return for ions undergoing MSAE, are shown with large circles. An analytic result, based on the Dehmelt and Hunter and McIntosh approximations is shown with a solid line in frame (b).

in Figure 5a, the strength of net axial force experienced by an ion increases dramatically with both radial and axial position outside of the $\left\langle E_{\mathrm{z}}\right\rangle_{\mathrm{RF}}=0$ cone. In consequence, the point at which axial ejection was assured should lie outside, but not very far outside, the cone defined by $\left\langle E_{\mathrm{z}}\right\rangle_{\mathrm{RF}}=0$. In fact, this condition is illustrated clearly by the data of Figure 11.
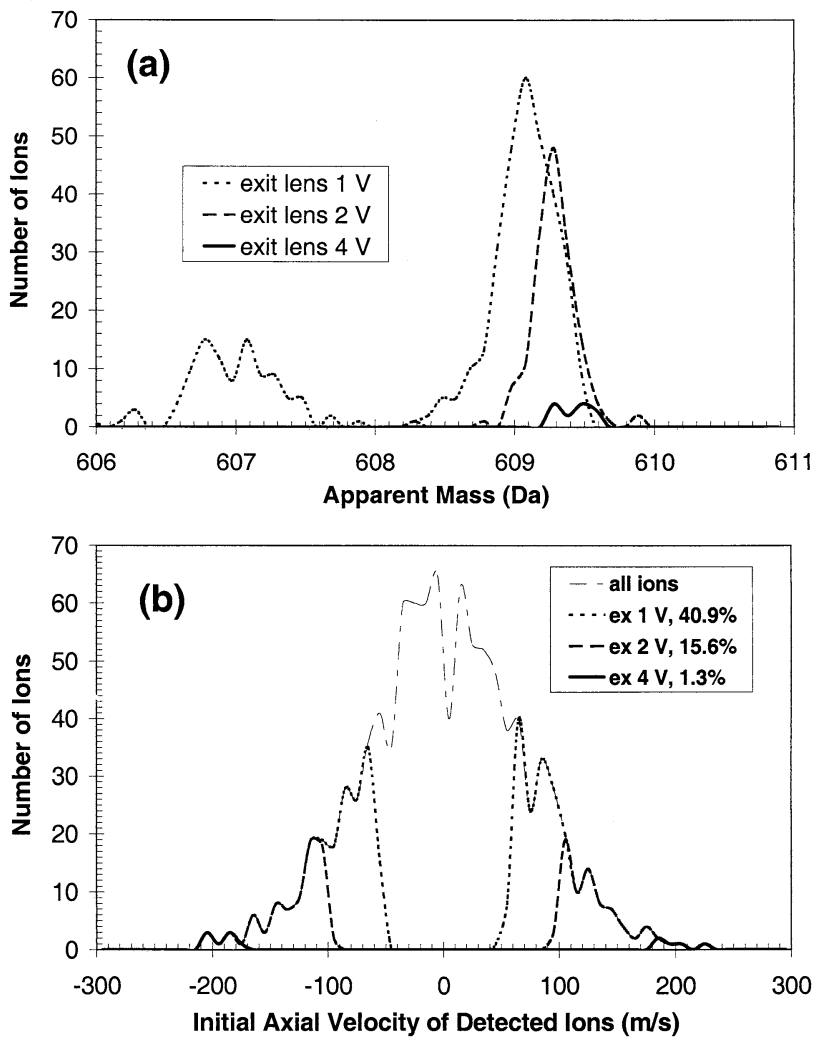

Figure 12. The simulated mass-intensity signals, corresponding to the data of Figure 11, that showed points of inflection in trajectory data at which axial ejection was assured, are shown in frame (a) with dotted, dashed and solid lines indicating exit lens potentials of 1,2 , and $4 \mathrm{~V}$, respectively. In all cases, the auxiliary amplitude was $0.2 \mathrm{~V}_{0-p}$. Frame (b) shows the distributions of the axial components of the initial velocities of those ions that were ejected axially, using the same line types to identify exit-lens potentials. The dot-dash line shows the total distribution of initial axial speed, consistent with thermal ions of mass $609 \mathrm{Da}$ and an ambient temperature of $300 \mathrm{~K}$. The percentages in the legends indicate the fractions of the initial populations that were ejected axially.

\section{Simulated Mass-Intensity Signals}

The simulated mass-intensity signals, corresponding to the data of Figure 11 that showed points of inflection in trajectory data at which axial ejection was assured, are shown in Figure 12a with dotted, dashed and solid lines indicating exit-lens potentials of 1,2 , and $4 \mathrm{~V}$, respectively. In all cases, the auxiliary amplitude was $0.2 \mathrm{~V}_{0-p}$. This auxiliary amplitude, combined with an exit-lens potential of $2.0 \mathrm{~V}$ yielded a good compromise between sensitivity and resolution. As expected from experiment, sensitivity decreased and resolution increased with increasing exit-lens potential. This result is consistent with Figure 11, which shows that, as the potential on the exit lens is increased, ions must approach the exit lens more closely before axial ejection is assured. Because the cone of reflection flares to larger radius at greater distance from the exit lens, ions that approach the cone with lower axial speed would require greater radial amplitude to be ejected axially. In addition, 
because ions are repelled by the exit-lens potential, it would be expected that only those, which approach it with some minimum axial speed, would be able to penetrate the cone.

This condition is illustrated clearly by the data of Figure $12 b$, which demonstrates that the axial speed, required to penetrate the cone, increases with the exitlens potential. Specifically, Figure $12 \mathrm{~b}$ shows the distributions of the axial components of the initial velocities of those ions that were ejected axially. The dot-dash line shows the total distribution of initial axial speed, consistent with thermal ions of mass $609 \mathrm{Da}$ and an ambient temperature of $300 \mathrm{~K}$. The dotted, dashed and solid lines correspond to similarly identified masspeaks of Figure 12a, corresponding to exit-lens potentials of 1,2 , and $4 \mathrm{~V}$. Integrated intensities of the mass peaks in Figure 12a, indicating ejection efficiencies, are shown in the legend of Figure 12b. In general, in the absence of collisions, thermal ions are reflected elastically by the exit-lens potential. Therefore, the distributions of the axial components of the ions' initial velocities remain representative of that condition until ions either penetrate the cone of reflection or are lost on the rods.

Parenthetically, the reader is reminded that to improve the ratio of ions, which were ejected axially, to those lost on the rods, during these simulations, ions were confined between two back-to-back fringing fields and MSAE was allowed at both ends of the device. Consequently, the efficiency of MSAE in these simulations is not representative of experiment and the efficiencies given, as percentages in the legend of Figure $12 \mathrm{~b}$, are intended for relative comparisons among specific values of the exit-lens potential.

\section{Experimental Mass-Intensity Signals}

Unfortunately, the authors are unaware of any suitable technique for mapping the cone of reflection experimentally. As a result, mass-intensity signals provide the only convenient point of comparison between this work and experiment. To this end, experimental data were obtained by selecting the ${ }^{12} \mathrm{C}$ isotope of reserpine in $\mathrm{Q} 1$, accumulating in Q3 and detecting the trapped ions by MSAE at a scan rate of $1000 \mathrm{Da} / \mathrm{s}$ using a dipolar auxiliary signal of $380 \mathrm{kHz}$ with constant amplitude 0.4 $\mathrm{V}_{0-p}$. The results are shown in Figure 13a where the dotted, solid and dashed lines correspond to exit-lens potentials of 2,4 , and $8 \mathrm{~V}$, respectively. Although these data are characteristic of good performance, there is nothing unusual about them.

Experimentally, the best compromise between sensitivity and resolution was achieved with auxiliary amplitude $0.4 \mathrm{~V}$ and an exit-lens potential of $4 \mathrm{~V}$. By contrast, when the trajectory calculations were collision-free, the simulator returned the best performance at roughly half these values. The lesser and greater exit-lens potentials of Figure 13a, as with Figure 12a, were chosen to demonstrate the consequences of halv-
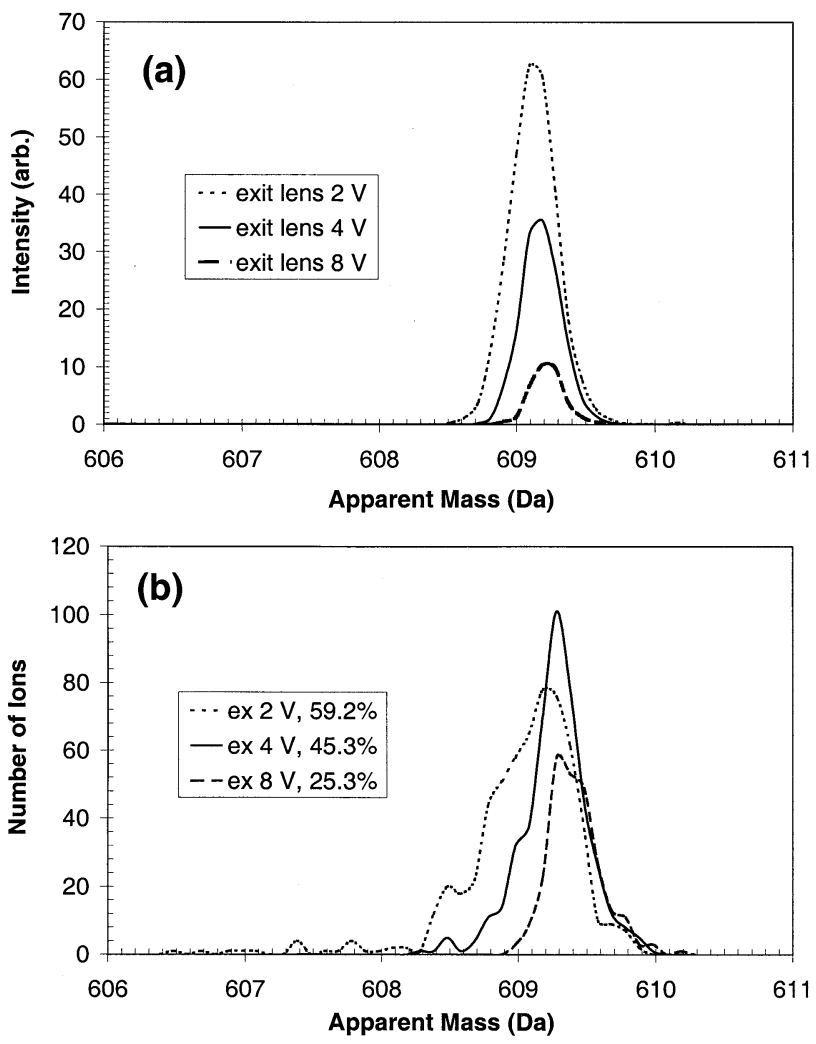

Figure 13. Mass-intensity signals of the 609 reserpine ion, collected at a scan rate of $1000 \mathrm{Da} / \mathrm{s}$ with the amplitude of the 380 $\mathrm{kHz}$ auxiliary signal held constant at $0.4 \mathrm{~V}_{0-p}$. The dotted, solid and dashed lines correspond to exit-lens potentials of 2, 4, and $8 \mathrm{~V}$, respectively. (a) Experimental. (b) Simulation results with ejection efficiencies indicated in the legend.

ing and doubling the exit-lens potential, which gave the best performance.

To obtain a more direct comparison with these experimental results, simulations were carried out in which ions moved freely through the entire length (133 $\mathrm{mm}$ ) of the Q3 cell and suffered collisions with nitrogen buffer gas at a nominal pressure of $3 \times 10^{-5}$ torr. As with the experimental data of Figure 13a, the mass range was scanned at a rate of $1000 \mathrm{Da} / \mathrm{s}$ using a dipolar auxiliary signal of $380 \mathrm{kHz}$ with constant amplitude $0.4 \mathrm{~V}_{0-p}$. The results are shown in Figure $13 \mathrm{~b}$ where the dotted, solid and dashed lines correspond to exit-lens potentials of 2, 4, and $8 \mathrm{~V}$, respectively. As before, MSAE was allowed from both ends to improve the efficiency of the simulations, so to compare with experiment, the ejection efficiencies in the legend must be halved. Choosing the optimal experimental conditions, of $0.4 \mathrm{~V}$ auxiliary amplitude with $4 \mathrm{~V}$ on the exit lens, the efficiency predicted by simulations for these conditions of $23 \%$ from Figure 13b, compares well with typical experimental values of about $20 \%$ [1].

In contrast to the collision-free simulations for which optimal auxiliary amplitude and exit-lens potential were below experimental values, it appears from a comparison of Figure 13a and b that when were collisions are enabled, optimal values for those parameters 
in the virtual instrument were greater than experimental. Specifically, in reference to Figure 13, the simulated mass peaks for exit-lens potentials of 4 and $8 \mathrm{~V}$ match most closely experimental results obtained with exitlens potentials of 2 and $4 \mathrm{~V}$, respectively. Despite this discrepancy, simulations demonstrate consequences similar to experiment when the exit-lens potential is varied about its optimal value. Specifically, when the exit-lens potential is too low, peaks are poorly resolved, with many ions being ejected too soon. As the exit-lens potential is increased, resolution improves but sensitivity diminishes. In addition, over a fairly broad range about the optimum value, the high-mass side of peaks are nearly coincident, while the low-mass side is shifted to higher mass with increasing exit-lens potential.

\section{Impact of the Amplitude of the Dipolar Auxiliary Signal}

The impact of the amplitude of the dipolar auxiliary signal, used for radial resonant excitation, was examined experimentally by holding the exit-lens potential constant at its optimum value of four volts and generating mass-intensity signals using auxiliary amplitudes both half and double the optimum value of $0.4 \mathrm{~V}_{0-p}$. As before, the ${ }^{12} \mathrm{C}$ isotope of reserpine was selected in Q1, accumulated in Q3 and detected by MSAE at a scan rate of $1000 \mathrm{Da} / \mathrm{s}$ using a dipolar auxiliary signal of 380 $\mathrm{kHz}$. Results obtained with auxiliary amplitudes of 0.2 , 0.4 , and $0.8 \mathrm{~V}_{0-p}$ are shown in Figure $14 \mathrm{a}$ with dashed, solid and dotted lines, respectively. Corresponding results, obtained from simulations using identical values for the exit lens potential and auxiliary amplitude are presented in Figure 14b. Otherwise, these simulations were similar to those used to obtain the data of Figure 13b.

In contrast to case of exit-lens potential, optimum auxiliary amplitude for these simulations appears to be somewhat lower than experimental. That is, the experimental results demonstrate the consequences of auxiliary amplitude that is too low most clearly, while the simulations illustrate the consequences of auxiliary amplitude that is too high most clearly. Even so, the consequences of varying auxiliary amplitude were similar in both regimes. When auxiliary amplitude was below optimum, ions gained radial amplitude more slowly and were ejected at higher apparent mass. In addition, resolution was degraded because ions of the same mass, which penetrated the cone of reflection, did so over a greater time period. Moreover, high-mass tailing resulted when ions retained sufficient radial amplitude to be ejected axially after they had passed through resonance, while at higher auxiliary amplitude, these ions would have been lost on the rods. In other cases, some ions passed through resonance without gaining sufficient radial amplitude to penetrate the cone of reflection at all and sensitivity was reduced.

Conversely, when auxiliary amplitude was too high,
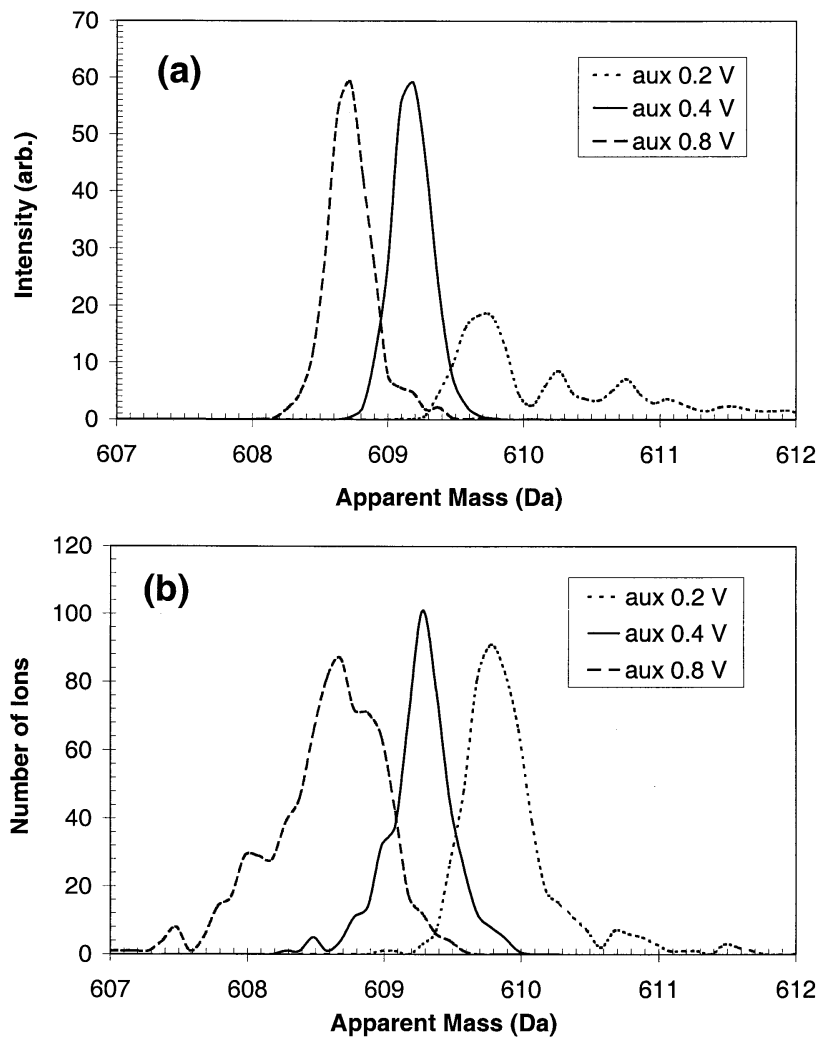

Figure 14. Mass-intensity signals of the 609 reserpine ion, collected at a scan rate of $1000 \mathrm{Da} / \mathrm{s}$ with the exit-lens potential held constant at $4 \mathrm{~V}$. The dotted, solid, and dashed lines correspond to amplitudes of the $380 \mathrm{kHz}$ auxiliary signal of $0.2,0.4$, and $0.8 \mathrm{~V}_{0-p}$, respectively. (a) Experimental. (b) Simulations.

ions gained radial amplitude more promptly and were ejected at lower apparent mass. The simulation results of Figure 14b show that resolution was degraded at higher auxiliary amplitude because some ions penetrated the cone of reflection too soon, as manifest by the low-mass tails on the lower-mass peak. Although not obvious from Figure 14, as auxiliary amplitude is increased further, sensitivity is reduced because many ions with lower axial speed are driven to the rods before they have an opportunity to penetrate the cone of reflection. These same consequences are observed both experimentally and in simulations, but require auxiliary amplitudes greater than those represented in Figure 14 to be manifest.

It is noteworthy that auxiliary amplitude, which is somewhat greater than optimal, can be compensated by increasing the exit-lens potential, but at the expense of sensitivity. In general however, performance is a stronger function of exit-lens potential, relative to the optimal value, than it is of auxiliary amplitude. These characteristics have been observed in both simulation and experiment.

Finally, as would be expected, and implicit in the discussion above, the cones of reflection, obtained from trajectory data of axially ejected ions, are unaffected by auxiliary amplitude when ejection- $q$ and exit-lens po- 
tential are held constant. Mass-intensity signals are affected strongly by auxiliary amplitude in consequence of the influence of the auxiliary signal on ion trajectories, not on the quadrupole and exit-lens fields, which determine the cone of reflection.

\section{Conclusions}

The diminution of the radial quadrupole potential in the fringing region near the end of a quadrupole rod array, gives rise to an axial electric field that can lead to mass-selective axial ejection of ions from a linear quadrupole ion trap. In consequence of their radial motion, which is characterized by extrema that are phasesynchronous with the local RF potential, the net axial electric field experienced by ions in the fringe field, over one RF cycle, is positive. To a first approximation, this axial field is proportional to the square of the radial displacement, the square of the stability coordinate $q$, and the mass-to-charge ratio of the ion.

When this axial field is superposed, in suitable proportion, with a static DC field due to a repulsive potential applied to the exit lens, a surface is defined in the fringing region on which the net axial force experienced by an ion over one RF cycle, is zero. In recognition of its shape, this surface has been dubbed the cone of reflection. Only ions with sufficient radial amplitude are able to penetrate this surface, and be ejected axially, while all others are reflected. Outside of the cone, ions experience a net positive axial force, which increases strongly with axial position. Inside, the relatively weak negative axial force diminishes in magnitude with decreasing axial position. Therefore, once an ion penetrates this surface, it feels a strong net positive axial force and it is accelerated toward the exit lens. Otherwise, the net axial force is negative and the ion is repelled. It could be said that the cone of reflection is the boundary between reflection and extraction regions, where the reflection region lies inside the cone of reflection. When the exit-lens potential is chosen judiciously, ions with thermal axial energies are able to penetrate this surface and be ejected axially only if they have sufficient radial amplitude. Ions lacking sufficient radial amplitude are reflected. In consequence, trapped, thermalized ions can be ejected axially, in a massselective way, by ramping the amplitude of the RF drive, to bring ions of increasingly higher mass into resonance with a single frequency auxiliary signal. In resonant response to the auxiliary signal, ions gain radial amplitude until they are either ejected axially or neutralized on the rods.

It may be possible to modify the cone of reflection by changing the nature of the fringing fields. The current simulation approach offers the opportunity to explore factors that change the MSAE efficiencies in a logical, step-wise fashion. The general trends of the simulations are in good agreement with experimental data, although there are discrepancies in the absolute magnitudes of the optimum auxiliary AC amplitudes and exit-lens potentials. Nonetheless, these simulations have provided needed clarification of the forces acting on ions exposed to quadrupole fringing fields that are not only important for mass-selective axial ion ejection from linear ion traps, but also for RF/DC and RF-only quadrupole mass spectrometers.

\section{Acknowledgments}

The authors thank Dr. Vladimir Baranov and Dr. Bruce Collings for helpful discussion and comments.

\section{Appendix A. The Sx32 Simulator}

\section{Overview}

The Sx32 simulator was developed in-house to provide an easy-to-use and easy-to-modify system, capable of simulating the trajectories of charged particles, which move under the influence of time-varying electric fields, suffer collisions with a buffer gas and interact electrically with each other. The simulator consists of three principal components: a user interface, ion generator and trajectory calculator. The user interface is written in Microsoft Visual Basic for Applications (VBA), compiled as an Add-In for Microsoft Excel. The ion generator and trajectory calculator are written in $\mathrm{C}++$ for the Microsoft Visual $\mathrm{C}++$ Compiler and are compiled and linked as dynamically linked libraries, DLLs, which become part of Excel at runtime.

In processes of interest, ions are created within (or introduced into) a particular device, their trajectories are manipulated through the application of electric potentials to electrodes, and finally, they are detected. This process, called a scan function, is composed of a series of segments. Each segment consists of a welldefined region, in either space or time, which can be described by a linear combination of specific mathematical models. For example, one segment may model a fringing-field input to a quadrupole rod array, the next a collision cell, the next a fringing field between rod arrays, etc. Often ions are detected during the final segment of a scan function in such a way that their mass-to-charge ratio can be measured.

Working in an Excel environment, the user can build ion populations which meet certain criteria, design and execute multiple-segment scan functions, examine the coordinate components of individual trajectories and energies, and extract statistically significant information, such as peak shapes, from a large number of trajectory calculations.

Collisions between ions and molecules of a neutral bath gas are of great importance in mass-spectrometric applications. Consequently, considerable effort has been devoted to the development of algorithms, which simulate realistic collision processes. The probability of collisions between ions and a neutral buffer gas can be calculated using either the Langevin or hard-sphere models, or a combination of both. Once a collision is 
deemed to have occurred, scattering can be modeled as either an elastic or inelastic process. The latter case includes the possibility of both exothermic charge exchange and endothermic fragmentation reactions.

\section{Trajectory Calculations}

The trajectory calculator obtains initial conditions for a specific segment directly from an Excel worksheet. The trajectory of the ion is calculated incrementally until a terminating boundary condition is met. Terminal conditions, for that segment, are written to the user interface and input for the next segment is prepared. This process continues automatically, until each ion has been processed by the scan function.

In all cases, forces on the ion are calculated by integration of the second order differential equation of motion

$$
\frac{\mathrm{d}^{2} \vec{r}}{\mathrm{~d} t^{2}}=-\frac{z e}{m} \nabla \Phi
$$

where $\vec{r}$ is the position vector of the ion, $z$ is the number and sign of elementary charges carried by the ion, $e$ is the electronic charge, $m$ is the mass of the ion, and $\Phi$ is the electric potential in three-dimensional space. When necessary, several mathematically distinct fields are superposed, simply by calculating the vector sum of the contributing forces.

Eq A1 is integrated numerically using Richardson extrapolation and the Bulirsch-Stoer method with adaptive step-size and error control. [Press, W. H.; Teukolsky, S. A.; Vetterling, W. T.; Flannery, B. P. Numerical Recipes in C, 2nd ed.; Cambridge University Press: Cambridge, 1992; Section 16.4, p 724.] In those cases for which the electric field is not known analytically, it is obtained by interpolating tabulated numerical solutions to the Laplace equation. The user can select an interpolation algorithm (polynomial, rational-function, or bicubic), which best suits the tabulated data. Although the bicubic algorithm works best with the integrator, it is the most complicated to implement and requires the pre-calculation and storage of derivative tensors.

\section{Solving the Laplace Equation}

Numerical solutions to the Laplace equation have been obtained, in both two and three dimensions, using Ansoft Corporation's Maxwell Field Calculators. The Ansoft package includes a CAD system, which is used to draw electrodes and define boundary conditions. Successively better solutions are obtained through both adaptive and manual refinement of a finite-element mesh. Mesh density, and the concomitant accuracy of the final solution, is limited by the amount of available RAM.

For most practical applications, the Ansoft fieldcalculator has provided solutions of acceptable accu- racy. One notable exception has been the weak axial fields in the fringing regions at the ends of quadrupole rod arrays. In instances such as these, where one field-component was several orders of magnitude weaker than those in perpendicular directions, the accuracy of the weak component was too low for practical use. The Ansoft code is not unique in this regard. In fact, it has returned solutions of comparable or higher quality than any other commercial code evaluated by the authors.

Recently, investigation of mass-selective axial ejection, MSAE, in which the weak axial fields in the fringing region at the end of a quadrupole rod array play a pivotal role, provided motivation to develop a Laplace solver in-house. The method of successive over-relaxation [Press, W. H.; Teukolsky, S. A.; Vetterling, W. T.; Flannery, B. P. Numerical Recipes in C, 2nd ed.; Cambridge University Press: Cambridge, 1992; Section 19.5, p 863] was adapted to this problem. In this application, electrode surfaces and symmetry boundaries were represented exactly and the potential calculated at regularly spaced grid points in three-dimensional space to a high degree of accuracy limited primarily by the precision of 8-byte reals.

\section{Differentiating the Potential}

Subsequent to solving the Laplace equation, the potential must be differentiated to obtain the electrical forces, which influence ion trajectories. Although several algorithms were evaluated, robustness, speed and ease of use made single-value decomposition [Press, W. H.; Teukolsky, S. A.; Vetterling, W. T.; Flannery, B. P. Numerical Recipes in C, 2nd ed.; Cambridge University Press: Cambridge, 1992; Section 15.4, p 671] the technique of choice. Implicit in single-value decomposition is the ability to reduce the noise that is inherent in numerical differentiation through a variable degree of smoothing.

Specifically, single-value decomposition was used to fit a polynomial to a relatively small number of points and the coefficients of that polynomial were used calculate the derivative at the midpoint of the segment. For example, to get the x-component of the electric field on grid points at which the potential was known numerically, a polynomial consisting of $\mathrm{m}$ terms was fit to $n$ sequential points, $n \geq m$, along a vector of constant $y$ and $z$. The coefficients so obtained were used to calculate the derivative at the single grid point at the midpoint of the segment. The starting point on the vector was incremented and the process repeated until derivatives at each grid point on the vector had been obtained. The degree of smoothing can be controlled by judicious choices for $\mathrm{m}$ and $\mathrm{n}$. Typically, an odd number of points, such as $n=5$, was chosen for the segment length. With $n=5$, the number of the terms in the polynomial would be chosen from the range $3 \leq \mathrm{m} \leq 5$. Choosing $m=3$ would provide significant smoothing of the potential and derived field components. With 
$m=5$, no smoothing of the potential would occur. The advantage of this strategy over the interpolation technique is that noisy fields can be smoothed and the degree of smoothing controlled by judicious choices for $m$ and $n$.

It was found that solutions to the Laplace equation, obtained by the method of successive over-relaxation, required no smoothing and the most accurate field components were obtained with $m=n$.

\section{References}

1. Hager, J. W. A New Linear Ion Trap Mass Spectrometer. Rapid Commun. Mass Spectrom. 2002, 16, 512-526.

2. Londry, F. A. "Mass-Selective Axial Ejection from a Linear Quadrupole Ion Trap. Proceedings of the 50th ASMS Conference, Orlando, FL, June, 2002; Poster MPM 377.

3. Schwartz, J. C.; Senko, M. W.; Syka, J. E. P. A Two-Dimensional Quadrupole Ion Trap Mass Spectrometer. J. Am. Soc. Mass Spectrom. 2002, 13, 659-669.

4. Hopfgartner, G.; Husser, C.; Zell, M. Rapid Screening and Characterization of Drug Metabolites Using a New Quadrupole Linear Ion Trap Mass Spectrometer. J. Mass Spectrom. 2003, 38, 138-150.
5. Dawson, P. H. Quadrupole Mass Spectrometry and Its Applications. AIP Press: Woodbury, NY, 1995; Chap V.

6. Prestage, J. D.; Dick, G. J.; Maleki, L. New Ion Trap for Frequency Standard Applications. J. Appl. Phys. 1989, 66, 1013-1017.

7. Melbourne, R. K.; Prestage, J. D.; Maleki, L. Analytic Potential in a Linear Radio Frequency Quadrupole Trap with Cylindrical Electrodes. J. Appl. Phys. 1991, 69, 2768-2775.

8. Dawson, P. H. Performance Characteristics of an RF-Only Quadrupole. Int. J. Mass Spectrom. Ion Processes 1985, 67, 267-276.

9. Hager, J. W. Performance Optimization and Fringing Field Modifications of a 24-mm Long RF-Only Quadrupole Mass Spectrometer. Rapid Commun. Mass Spectrom. 1999, 13, 740748.

10. Thomson, B. A.; Jolliffe, C. L. Spectrometer with Axial Electric Field. U.S. Patent No. 5 847,386, 1998.

11. Dawson, P. H. Quadrupole Mass Spectrometry and Its Applications. AIP Press: Woodbury, NY, 1995; Chap II and III.

12. Dehmelt, H. G. Radiofrequency Spectroscopy of Stored Ions I: Storage. Adv. Mol. Phys. 1969, 3, 53-72.

13. Hunter, K. L.; McIntosh, B. J. An Improved Model of the Fringing Fields of a Quadrupole Mass Filter. Int. J. Mass Spectrom. Ion Processes 1989, 87, 157-164.

14. March, R. E.; Hughes, R. J. Quadrupole Storage Mass Spectrometry, Chemical Analysis 102. John Wiley and Sons: New York, NY, 1989; Chap II. 